\title{
Making regulation fit by taking irrationality into account: the case of the whistleblower
}

\author{
Sebastian Oelrich ${ }^{1}$ (iD
}

Received: 4 December 2017/ Accepted: 26 February 2019/Published online: 7 March 2019

(C) The Author(s) 2019

\begin{abstract}
Prospect theory describes people as bounded rational decision maker. What sparked widespread discussion after its initial introduction in 1979 is today criticized for lack of applicability. I use the debate about whistleblowing laws to show that prospect theory may be applied prescriptively in economics as a tool to design effective legislation. Whistleblowing is often seen as an important way to uncover fraud, which causes billions of USD in damages annually. I first examine the fragmented legal landscape across Europe, showing that it can be framed as one favoring rewards or the prevention of losses. I conduct an experiment with 39 university students, wherein legislative incentives are evaluated under a prospect theoretical frame in a setting of ambiguity and high stakes. Results suggest that people exhibit the typical s-shaped value function and loss aversion in line with prospect theory. In addition, their intention to whistleblow is more heavily reduced by losses than increased by gains. The study adds to the scarce literature of prospect theory on decisions in ambiguous contexts - as well as to the applicability of the theory as a prescriptive instrument in designing institutional frames. For whistleblowing in particular, a protection-based approach seems most promising.
\end{abstract}

Keywords Fraud · Loss aversion · Prospect theory · Regulation · Whistleblower

JEL Classification D81 - D91 $\cdot$ K22 $\cdot$ M48

Sebastian Oelrich

sebastian.oelrich@ovgu.de

1 Chair of Business Economics and Accounting, Otto-von-Guericke-University Magdeburg, Postbox 4120, 39016 Magdeburg, Germany 


\section{Introduction}

Prospect theory has garnered widespread attention after its first introduction in 1979 by Kahneman and Tversky. However, "it is curious, then, that so many years after the publication of the 1979 paper, there are relatively few well-known and broadly accepted applications of prospect theory in economics" (Barberis 2013, p. 173). Tversky and Kahneman (1992, p. 317) explain this lack of applicability by stating that "rationality in economic theory is commonly justified [...] [by] the fear that any treatment that abandons rationality will be chaotic and intractable" (Tversky and Kahneman 1992, p. 317). Although there are some applications especially in management and finance literature that use prospect theory to explain observed behavior, it is rarely used as a prescriptive tool (Barberis 2013). In this paper I show a first solution to this issue as I use prospect theory to evaluate laws that explicitly work in a dichotomous framing of gains and losses. Whistleblowing laws are used as an example to show the applicability in a legal economics context that need not only be descriptive, but should indeed be prescriptive, resulting in more effective laws.

Governments and companies have long sought to bring about successful measures to detect cases of economic crime. Accounting departments are scrutinized by external auditors to assure investors of compliant behavior with law. However, large scandals at companies such as Enron, WorldCom, Siemens, and Deutsche Bank occurred despite these actors (Ball 2009). According to the Association of Certified Fraud Examiners (ACFE), the average firm loses 5\% of revenue to fraud annually, amounting to 3.7 trillion USD worldwide (ACFE 2016) — without taking into account the costs for loss of reputation, trust, and efficiency due to fraudulent behavior ( $\mathrm{Li}$ and McNeil 2006; Charreire Petit and Cusin 2013).

Fraud is most frequently detected by non-traditional actors, such as employees (Dyck et al. 2010; ACFE 2016). Whistleblowing therefore is an essential tool in uncovering fraud within private and public institutions (Wilde 2017; Call et al. 2018). It can be utilized not only to uncover (reactive), but also to prevent (proactive) such fraudulent behavior, preventing much of the negative consequences (Bussmann 2015; Cordis and Lambert 2017). While whistleblowing is shown to be an effective tool, it is still being underutilized by much of economics (Villena and Villena 2010). Whistleblowers often experience retaliation, especially in countries without whistleblowing laws (GBES 2016). In Europe for example, only half of the countries have such laws in place. In addition, these are rooted in moral arguments, instead of being evaluated by their effectiveness in an economic frame (see part 2.2).

I start by evaluating whistleblowing laws across Europe, taking the recent initiative for a directive proposal by the European Commission as a starting point. I find that only half of the countries have designated laws in place, where a recurring theme is the protection from retaliation and a clear lack of a reward structure. One of the few countries worldwide, the USA has introduced a comprehensive reward scheme for whistleblower with the Dodd-Frank Act in 2010, after previously 
providing protection to whistleblower with the introduction of the Sarbanes-Oxley Act in 2002. This clear dichotomy between rewards or bounties and protection from losses, e.g., retaliation or sanction, is a recurring theme in whistleblowing laws. While much whistleblowing research has focused on individual and situational factors (Lee and Xiao 2018), few have taken laws specifically into account. What has so far, to my knowledge, not been done is an evaluation of the effectiveness of such laws using prospect theory.

I execute a laboratory experiment with 39 university students with an economic background. In a first stage, each participant's value function is assessed in a whistleblowing case under high stakes and ambiguity. This is done using a method recently developed by Abdellaoui et al. (2016) that makes the entire value function in prospect theory observable. In a second stage, their general whistleblowing intention and their specific changes in intention according to different situations and prospects based on their answers in the first part are assessed.

The results show that the majority of participants exhibit the typical s-shaped value function as proposed by prospect theory. They are risk seeking (averse) in the domain of losses (gains) and show a convex (concave) value function. This also implies that framing is important, as people evaluate outcomes in terms of gains and losses from a neutral reference point (reference dependence). They show loss aversion of a magnitude similar to prior studies for risk and uncertainty. The value function and the loss aversion are not correlated to whistleblowing intention, which provides further proof that this is a typical behavioral trait (Tversky and Kahneman 1992). However, participants showed stronger intention decrease when faced with a loss prospect of the same value-magnitude change from the reference point than for an increase in intention for a change toward a gain. In addition, an uncertain loss decreases whistleblowing less than a certain loss of the same value, whereas there is no difference between a certain and an uncertain gain. Participants show the typical traits proposed by prospect theory that losses loom larger than gains and therefore a loss impacts their intention to whistleblow stronger than a gain. A result of this finding is that mitigating the loss side of the value function should be the priority in any legislative effort for whistleblowing. That is, a protective scheme, even if it does not fully or certainly protect, is more relevant in the decision process than a significantly higher gain.

A more generalized implication of this finding is the application to incentive systems in law overall. Prospect theory can provide valuable insights into how people can be (dis)incentivized to take action. Designing legislation with this knowledge in mind can further increase the effectiveness of a legislative effort, especially when outcomes can be framed as losses and gains. For our understanding of prospect theory, this paper provides insight into the application for uncertainty (ambiguity) and high stakes, where literature is still scarce (Abdellaoui et al. 2016). It shows that prospect theory holds for situations under uncertainty, as well as high (hypothetical) outcomes.

The rest of the paper is structured as follows. Part two describes whistleblowing (research) and gives an overview on the scattered legislation across Europe. I then briefly describe prospect theory and relate my research to the literature, addressing shortcomings and issues of applicability. Hypotheses are developed from the discussion here. The fourth part contains the experimental setup and execution. The 
fifth part presents the results. The last part discusses the findings and avenues for future research.

\section{Whistleblowing and the law}

\subsection{Whistleblowing research}

Near and Miceli (1985, p. 4) describe whistleblowing as a "disclosure by organization members (former or current) of illegal, immoral or illegitimate practices under the control of their employers, to persons or organizations that may be able to effect action." This definition is used widely across academic literature. Whistleblowing is characterized as a highly complex decision process (Chiu 2003), including a multitude of situational, individual, as well as institutional factors (Near and Miceli 1996; Mesmer-Magnus and Viswesvaran 2005; Lee and Xiao 2018). Although no formal model has emerged as of today (Lee and Xiao 2018), the general structure of the process can be characterized as shown in Fig. 1. The internal agent observes what might or might not be an actual case of fraud by another person

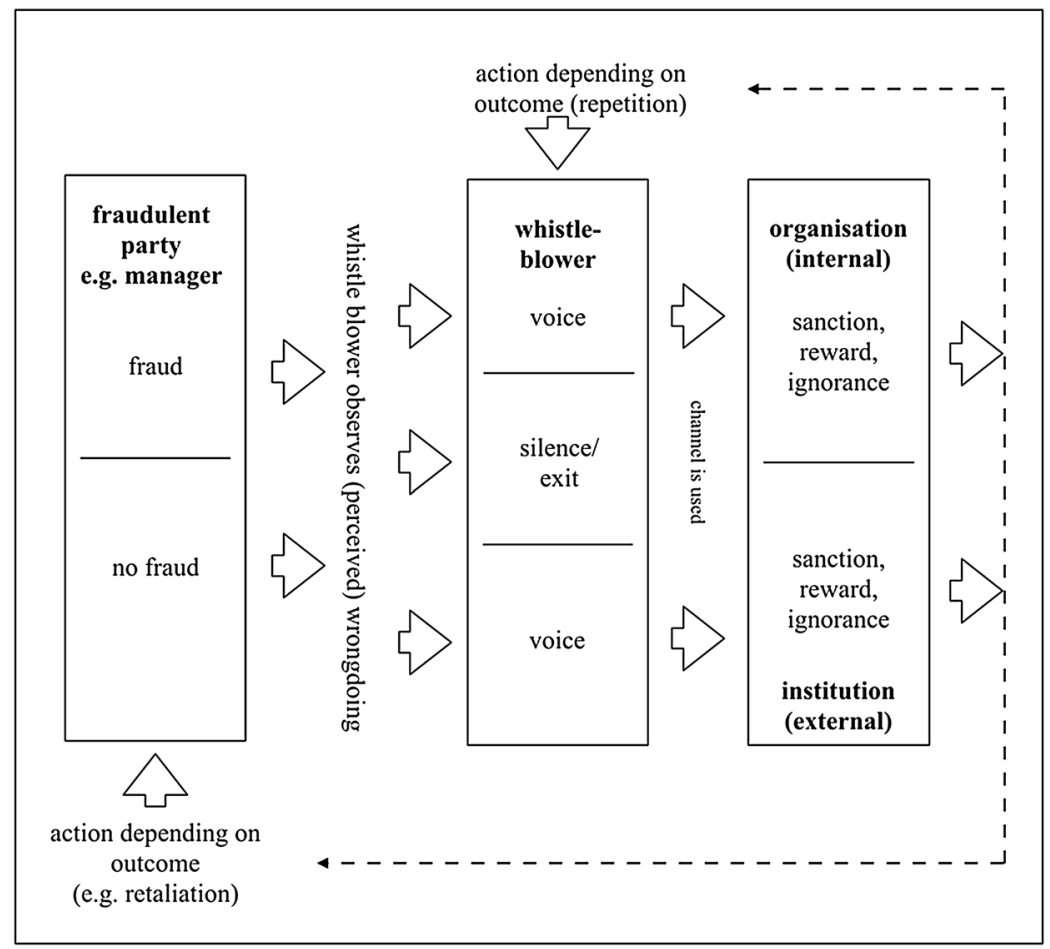

Fig. 1 Whistleblowing process where the potential whistleblower observes fraud and can choose between voice, silence, and exit, which evokes reactions by other internal or external parties of rewards, sanctions, or ignorance 
of the organization. The potential whistleblower can now choose to speak up, thus exercising "voice", remain "silent", or leave the organization, choosing "exit" (Hirschman 1970). This decision is highly dependent on various factors of individual, situational, and institutional nature. Choosing any other option but "voice" ends the process, and the agent never becomes a whistleblower. Choosing "voice" instead, there are two options for her to choose from in the next stage of the process. She can blow the whistle either internally or come forward to an external channel. ${ }^{1}$ The disclosure of information to any third party will evoke one of three reactions by that party: sanction, reward, ignorance, or a combination thereof (Near and Miceli 1985). Depending on the outcome, the whistleblower will either accept or return to the previous stage, repeating the process. For example, in the case of internal whistleblowing: after no action followed the disclosure, the whistleblower might be compelled to take her claims outside of the organization (Callahan and Dworkin 1994; Gray 2004). At the same time, the person accused of the fraudulent act might be compelled to take actions if she becomes aware of the disclosure and the whistleblowers' identity.

To understand this process and especially the influential factors has been at the core of academic research for decades. Research in the field of social sciences and behavioral economics started with individual and situational factors such as gender, age, occupation, and type of wrongdoing (Miceli and Near 1984; Near and Miceli 1985; Dworkin and Baucus 1998). The last two decades saw a rise in studies concentrating on institutional factors such as culture as defining characteristics. Mixed results suggest that the influence of culture cannot easily be depicted (Sims and Keenan 1999; Keenan 2002, 2007; Park et al. 2008; Cheng, Karim and Lin 2015). Literature outside of the USA on this topic is scarce, which is why scholars argue that research originating from other countries is necessary to advance our understanding of the whistleblowing process (Vinten 2004; Brown et al. 2014; Gao and Brink 2017).

\subsection{Regulation overview}

It is usually the USA that is associated with strong whistleblowing laws. The Enron scandal can be seen as a starting point in the development of laws for whistleblower, although the False Claims Act of 1986 already rewarded people who spoke out externally when the government was defrauded by a private entity (Callahan and Dworkin 1994). After the famous Enron case and the subsequent collapse of auditing firm Arthur Andersen, the Sarbanes-Oxley Act 18 U.S.C. $§ 1514$ A (SOX) was introduced in 2002 as part of a reformed capital market regulation (Dworkin 2007). Members of the organization in which the alleged fraud such as bank fraud, securities fraud, or any breach of regulation of the Securities and Exchange Commission (SEC) occurred, may report to a designated third party (superior, congress member, SEC). The plaintiff (whistleblower) is entitled to compensation for discharge or discrimination following the disclosure (SOX 18 U.S.C. $\S$

\footnotetext{
1 Examples for internal channels are ombudspersons, talking to management, or specifically established e-mail addresses or hotlines, while external channels might be designated agencies, media, or the public.
} 


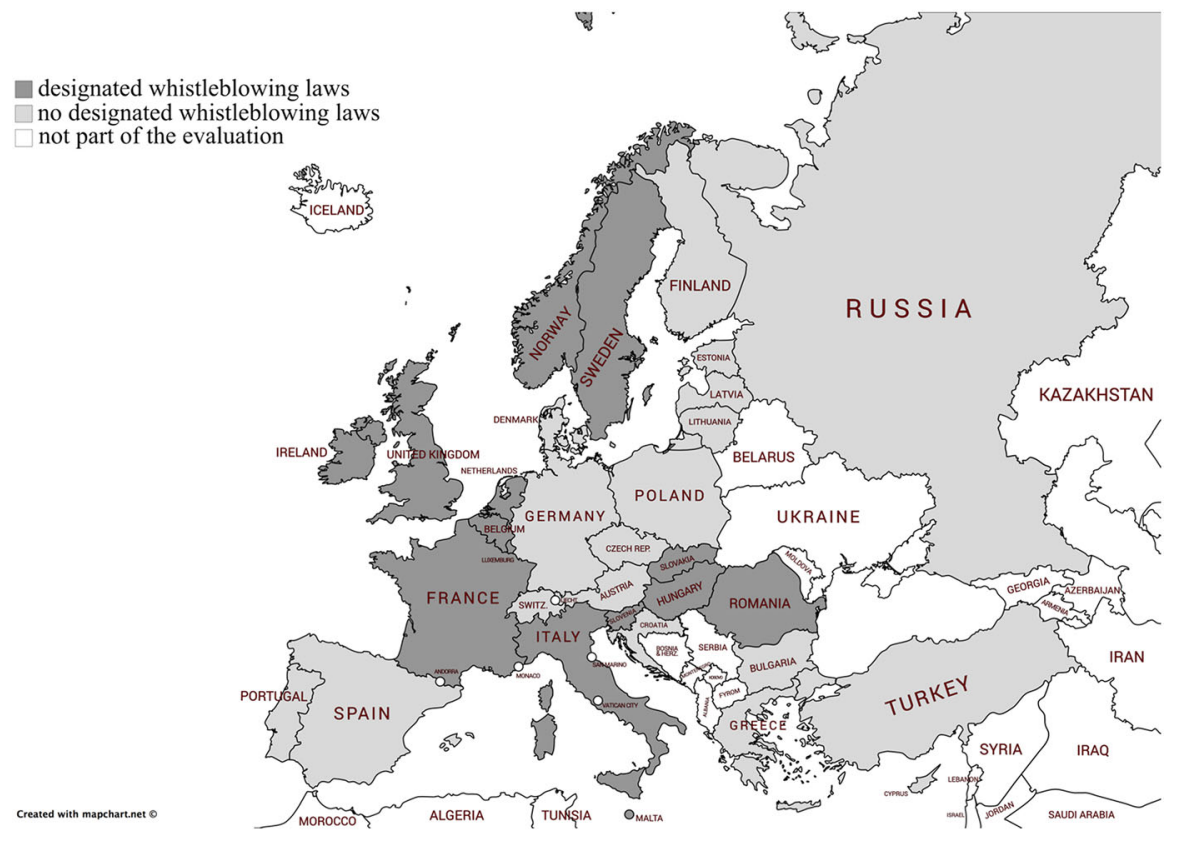

Fig. 2 Overview of whistleblowing laws in Europe and select other countries

1514A(a)). Scholars such as Dworkin (2007) argue that the protection is inefficient and unlikely to increase reports. Moberly (2007) shows that in the first 3 years after the introduction of SOX only 3.6\% of whistleblower won relief through the initial process, concluding that SOX is misapplied to the disadvantage of whistleblower.

The Dodd-Frank Wall Street Reform and Consumer Protection Act 15 U.S.C. §78u-6 (DFA) was passed in 2010. While restricting the capital markets after the financial crisis, Section 922 provides additional regulation in relation to SOX. The DFA overlaps with the SOX in some regards of protection but introduces a rewardbased incentive for whistleblower (15 U.S.C. \$78u-6(a)). Tips to the SEC concerning violations of securities laws resulting in successful actions against the person or organization charged may be compensated, if the monetary sanctions recovered from the convicted party exceed one million USD. Then the whistleblower is eligible to a reward between 10 and 30\% (15 U.S.C. $\$ 78 u-6(b)$ also in general Mogielnicki 2011).

Europe, on the other hand, can be separated in two equally large camps. The first one does not have designated whistleblowing laws, while the second does. Figure 2 shows a map where countries within the European Union and adjacent ones of particular interest are classified according to their whistleblowing laws. ${ }^{2}$

\footnotetext{
${ }^{2}$ A detailed list of where to find the specific law for each country is given in Appendix 1. Whenever possible, a translated version of the law was used.
} 
Those countries that do not have designated laws usually have some degree of protection scattered across other laws, most prominently labor law. Internal (formal) channels to go to with information do exist unevenly, and often in the public sector only, probably to combat corruption.

A reason why some countries have whistleblowing laws, while others do not may be found in their attitude toward that behavior. In Germany for example, a word often used in connection with whistleblower is "Denunziant." It has a strong negative connotation and can most accurately be translated as tattle-tat or squealer (Rauhofer 2007). The perception of whistleblower in Germany is strongly influenced by history, especially Nazi-Germany and later again in the former East, the GDR. Here, spying on neighbors, friends, colleagues, and family was encouraged and even demanded by state authorities such as the Stasi and Gestapo (Gibeaut 2006; Rauhofer 2007). This negative view on whistleblowing is still very much alive, as recent surveys suggest. Bussmann et al. (2013) find that of companies that have a whistleblowing system established, $48 \%$ believe that it fosters "squealing." A similar history shaped the negative view in South Africa. In India, whistleblowers are under threat to be killed (Gibeaut 2006). This negative view might also be a factor in other countries that do not have whistleblowing laws in place. In countries like the USA, on the other hand, that do have whistleblowing laws, they are sometimes referred to as heroes (Grant 2002; Hartman et al. 2009).

Countries that do have designated laws are shown in more detail in Table 1. Most laws are relatively new, passed within the last decade. An exception is the UK. The Public Interest Disclosure Act (PIDA) enacted in 1998 provides employees with protection against retaliation if they make a disclosure in the public interest. However, the law is often characterized as lacking real protection from retaliation, being flawed and inefficient (Bowden 2006; Latimer and Brown 2008). Many countries abandon the "in good faith" provision in whistleblowing laws in favor of a "in public interest" definition. The test whether a whistleblower is protected under the law is therefore less strict: as long as the disclosure is made under reasonable belief of a wrongdoing that is thought to harm the public. The "in good faith" test, on the other hand, often led to courts waiving protection eligibility (Dworkin 2007). Most laws state that internal channels have to be exhausted before making external disclosures. Only a handful of countries allow the public and media as valid external recipients. Ireland is the only country that could — at least indirectly-be considered as having a reward provision. Whistleblower may receive a compensation up to 5 times their annual salary. However, that compensation is only paid in an unfair dismissal case and reduced if the intention was not made in good faith.

In April 2018, the European Commission published a draft directive for the European Parliament on the protection of whistleblower (European Commission 2018). This directive would be in line with international best practice, as it does enable whistleblower to report the misconduct externally even to media and the public. It would be a purely protection-based legislation, which prohibits any form of retaliation if the whistleblower reported the incident to the best of her knowledge and the disclosure is in the public interest.

The effects of legislation have been analyzed more prominently after the introduction of the Sarbanes-Oxley Act in 2002 and mostly with a focus on the 


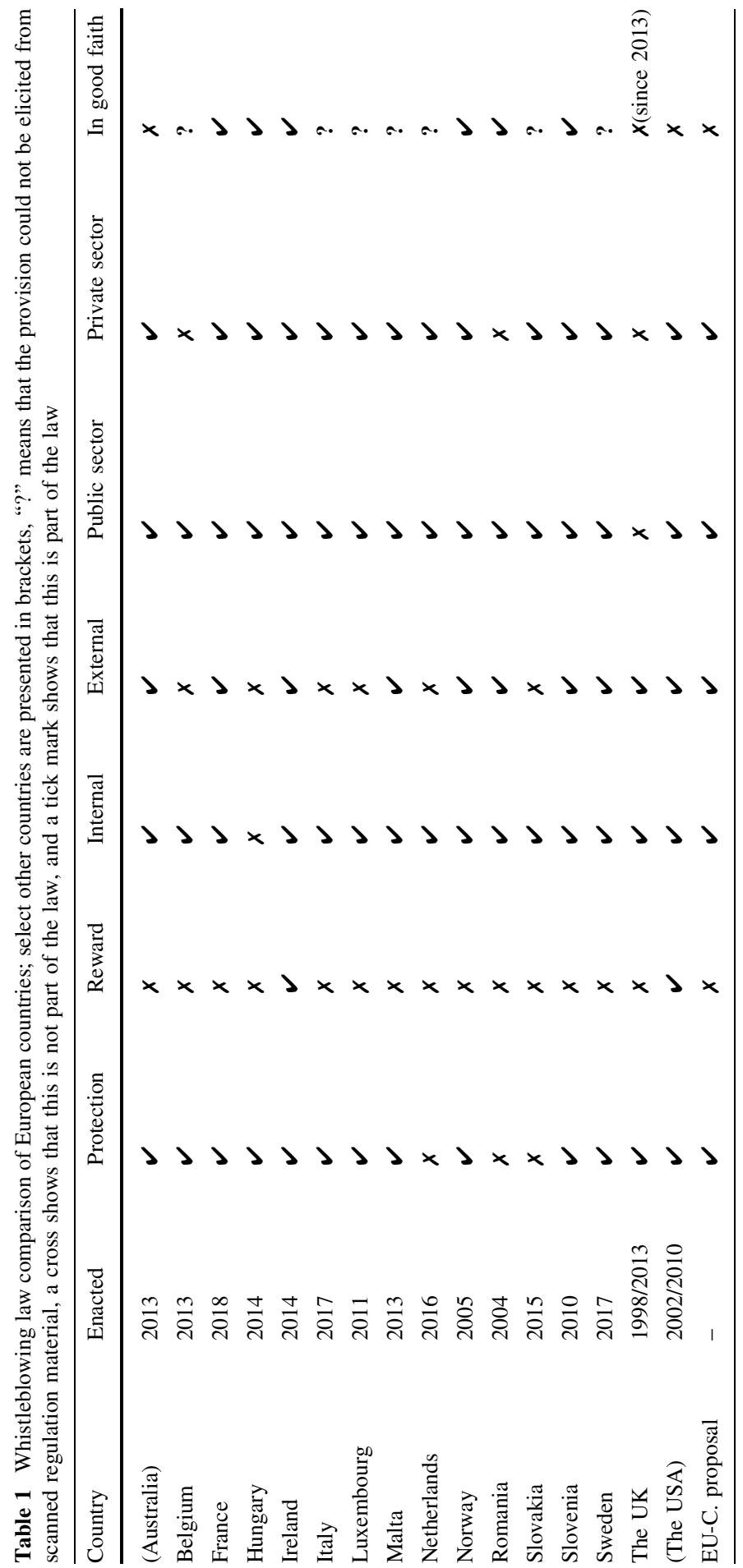


USA. Dworkin (2007) doubts that SOX would foster whistleblowing due to its ineffective protection. Moberly (2007) shows that only $3.6 \%$ of whistleblower won relief after experiencing retaliation through SOX within the first 3 years after its introduction. This is emphasized again by Yeoh (2014) who argues that neither the US (SOX and DFA), nor the UK's PIDA brings actual whistleblower protection. Schmidt (2005) argues that rewards and protection granted for external whistleblowing may lead to opportunistic behavior that reduces organizational efficiency. He concludes that laws such as the UK's PIDA or the SOX (and in extension the then not yet introduced DFA) cannot claim legitimacy. Experimentally, Brennan and Kelly (2007) show that protection increased external whistleblowing intention for accounting students in Ireland, while Perreault and Wainberg (2016) find that explicit protection statements in internal channels (hotlines) increase the perceived threat to retaliation and thereby decrease whistleblowing intention.

Mogielnicki (2011) argues that the extension by the DFA would incentivize whistleblower by utilizing rewards for external whistleblowing. This is in line with the experimental findings by Andon et al. (2018) who show with a group of professional auditors that financial rewards lead to higher external (e.g., authorities) whistleblowing intention. However, this effect is mediated by the seriousness of the wrongdoing. That is, rewards are less significant when the seriousness is high enough. However, Stikeleather (2016) only finds a positive influence on internal whistleblowing. Similar results by Brink, Lowe and Victoravich (2017) show that monetary attitude leads to increased whistleblowing intention, although they find no difference between internal and external channels. Rose et al. (2018) only find an influence for very high rewards. A different perspective is given by Cordis and Lambert (2017) who find that whistleblowing laws have a deterrent effect on fraud, which they attribute to the higher perceived detection rate by companies or (potential) wrongdoers.

This analysis shows that the dichotomy of "rewards vs protection" is a recurring theme in legislation and academic research. It is evident that people seem to evaluate whistleblowing laws within these two frames. This is paramount when prospect theory is applied on this issue in the subsequent chapter. In particular, a protective approach aims to minimize or prevent retaliation. The influence of retaliation on whistleblowing has long been studied. Most research finds that whistleblowing intention is reduced by an increase in retaliation likelihood or severity (e.g., Miceli and Near 1984; Mesmer-Magnus and Viswesvaran 2005; Liyanarachchi and Newdick 2009; Mayer et al. 2013, Caillier 2017; Dhamija and Rai 2017). Interestingly, Perreault and Wainberg (2016) find that explicit protection statements in internal channels (hotlines) increase the perceived threat to retaliation and thereby decrease whistleblowing intention. ${ }^{3}$

\footnotetext{
3 For further insights, Gao and Brink (2017) and Lee and Xiao (2018) provide comprehensive overviews on whistleblowing, including retaliation aspects for previous whistleblowing studies.
} 


\section{Prospect theory and hypotheses development}

\subsection{Prospect theory recap}

Prospect theory was introduced by Kahneman and Tversky in 1979 as a descriptive model of decisions under risk. It gives experimental evidence that people tend to value changes in terms of deviations from a reference point (reference dependence), for example a status quo. From this point, deviations can be classified into gains and losses, where people tend to be risk averse toward gains and risk seeking toward losses, while the latter effect is more pronounced than the former and is called loss aversion (Kahneman and Tversky 1979). Contrary to what economists usually refer to as the "homo oeconomicus", Kahneman and Tversky find that instead of being purely rational, people can more accurately be described as bounded rational. However, their actions are not completely irrational, that is, they do evaluate options within constant frames and patterns (Kahneman and Tversky 1979, Tversky and Kahneman 1992). While this original version was limited in several regards, a revised version was introduced in 1992, solving issues of stochastic dominance violations, the limitation to two nonzero outcomes that could be examined, as well as extending the theory from decisions under risk to uncertainty. In addition, decision weights can now be assessed separately for gains and losses (Tversky and Kahneman 1992).

The generally known function of expected utility is the sum of probability $p$ of an outcome multiplied by the outcome value $x$ for all outcomes, where the added probabilities are 1 . In prospect theory this function is altered in several regards. A value $v$ is assigned to an outcome $x: V(x)$, and a decision weight $w$ is assigned to the probability $p$ of an outcome: $W(p)$. The decision weight may vary for gain and loss prospects, respectively, thus $w^{+,-}(p)$. A typical value function under prospect theory is shown in Fig. 3. The laws introduced in the previous chapter are added to this value function according to their influences: Protection-based legislation is added to the loss (prevention) side and rewards are added to the gain side of the value function.

The origin can be interpreted as the status quo or the neutral reference point. The function for positive (negative) outcomes is concave (convex), and the function for losses is steeper than for gains, depicting loss aversion. The value function in parametric form can be written as

$$
v(x)\left\{\begin{array}{cc}
x_{j}^{\alpha} & x \geq 0 \\
-\lambda\left(-x_{j}\right)^{\beta} & x<0
\end{array},\right.
$$

where $\lambda$ is the parameter signifying loss aversion, if $\lambda>1$. This "new version" (1992) has been shown to give better results in regard to explaining human behavior not only compared to the original version (Fennema and Wakker 1997), but also in comparison with other versions of utility descriptions (Kothiyal et al. 2014).

Prospect theory's introduction has sparked numerous academic researches that try to apply it to economic problems. Most notably in the area of management research, especially strategic management, organizational behavior, and human 


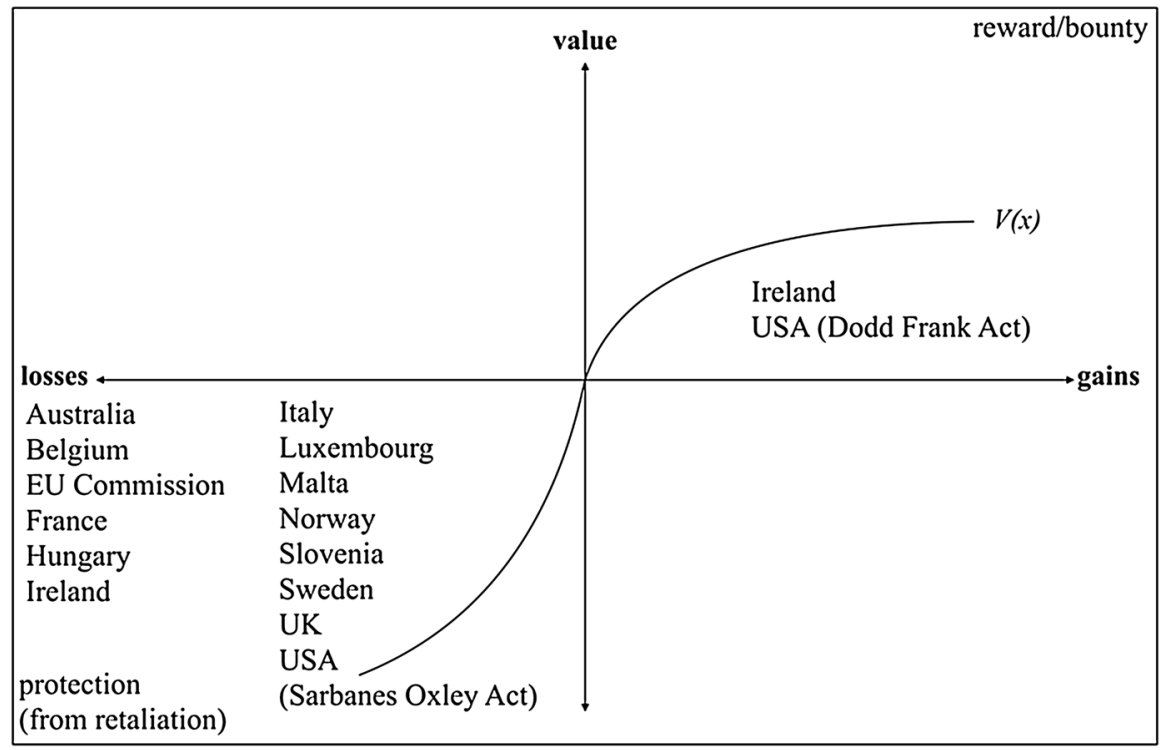

Fig. 3 Typical value function in prospect theory where an outcome $x$ is weighted by a value $v$ and the origin represents the reference point or status quo; legislation is added to the corresponding quadrant

resource management, targeting executive compensation, negotiations, relations between organizational risks and return and firm risk-taking behavior. However, Holmes et al. (2011) find in their review of these studies that most either misconstrue prospect theory or only apply aspects of it on the issue. Outside of management, an obvious application is the behavior of people in finance, in relation to portfolio and investment decisions and insurance contexts, especially insurance schemes and preferences (for a review see Barberis 2013). More recent studies try to extend these applications to other areas. Yang et al. (2017) use prospect theory to explain traveler's choices under travel time variability. Weingarten et al. (2018) use it to show that a positive deviation in one area (examinations) does not compensate for a failure in another area. However, research mostly uses prospect theory to describe observed behavior, instead of using it in a prescriptive way (Barberis 2013). Its application lack outside of pure economic contexts is staggering. Thus, the actual impact of prospect theory is still out for debate, even 40 years after its initial introduction.

\subsection{Reference point discussion}

One reason for lack of applicability may be due to the vague definition of the reference point (Köszegi and Rabin 2007; Barberis 2013). Literature refers to it mainly as the current wealth or status quo (Kahneman and Tversky 1979; Samuelson and Zeckhauser 1988; Tversky and Kahneman 1992), as expectations (Cherry et al. 2003; Köszegi and Rabin 2007) or goals (Weingarten et al. 2018). For whistleblowing in particular, it may be interpreted as expectations about what would 
happen under "normal" circumstances, a continuation of the current state of nature, that is expectations about the future: The person goes to work and does her job. To discover a fraud case is most likely unexpected or unanticipated. The subsequent consequences of going public with that information are a deviation from the expectation about the future. It involves a deviation from the expected or current wealth, e.g., her salary and employment position.

\subsection{Risk and ambiguity}

A second reason for the lack of applicability might be the common understanding of prospect theory as decision under risk - and not under uncertainty, which captures a wider variety of actual problems (Kothiyal et al. 2014). Originally, prospect theory was devised as a theory to describe decisions under risk. However, due to Tversky and Kahneman's (1992) extension of prospect theory, it is able to capture situations under uncertainty, also called ambiguity in the Knightian sense (Knight 1921). Ellsberg (1961, p. 657) famously showed that people prefer risk over ambiguity, which he defines as "a quality depending on the amount, type, and 'unanimity' of information, and giving rise to one's degree of 'confidence' in an estimate of relative likelihoods." However, as Kothiyal et al. (2014, p. 2) lament, "there is still a widespread misunderstanding that prospect theory could only be applied to risk. The popularity of prospect theory for risk may have contributed to this misunderstanding," which may be one of the reasons why there are so few experiments of prospect theory under ambiguity. Notable exceptions are Kothiyal et al. (2014), Abdellaoui et al. (2016), and Baltussen et al. (2016), which I will refer to in the experimental section.

Whistleblowing seems to be better described by a situation under ambiguity than under risk. Risk proposes that the probabilities within a prospect are known. However, this is highly unlikely for a whistleblowing scenario_-or any real-life problem. For example, the US SOX on paper gives a $100 \%$ protection from retaliation if certain conditions are met. Moberly (2007) shows that only $3.6 \%$ of whistleblower won relief. The US SEC awarded rewards to 46 individuals between 2012 and 2017 only, even though they received over 4400 tips in 2017 alone (SEC 2018). Many of these whistleblowers probably genuinely believed they would be eligible for a reward. This contributes to the interpretation that probabilities are unknown or hard to estimate. Every fraud and whistleblowing case is unique, and comparing one's own case to that of another whistleblower is problematic. In this paper, the situation is therefore interpreted as a decision under ambiguity. In addition, this helps to advance our understanding of the validity of prospect theory for ambiguous contexts and adds to the scarce experimental literature in this area.

\subsection{High stakes and payoffs}

A third issue is whether prospect theory is a laboratory phenomenon or an actual behavioral trait (Tversky and Kahneman 1992; Barberis 2013). Laboratory experiments often use low stakes and small amounts of money, while they are also hypothetical decision problems and mostly restricted to monetary outcomes. 
However, it has repeatedly been shown that this way of decision making stays constant across different problems, such as decisions involving monetary outcomes (Hershey and Schoemaker 1980) as well as human lives (Tversky and Kahneman 1981). Kachelmeier and Shehata (1992) show that there are no significant differences between hypothetical and real payoffs, or large and small amounts of money. Studies found no significant differences between a flat compensation of the participants in the experiments and a payoff-based pay (Camerer 1989, Kachelmeier and Shehata 1992). However, literature on large amounts of money remains scarce, a point where this study aims to provide further insights, using high (hypothetical) payoffs that seem more realistic in the whistleblowing context (rewards: SEC 2018; losses: GBES 2016).

Prospect theory seems very much suited to evaluate whistleblowing decision making, considering the framing of legislation in terms of losses (protection) and gains (reward). Each whistleblowing case is highly individual, and therefore, outcomes are ambiguous. The reference point as the expectation of the future or status quo and the detection of fraud as an unexpected deviation seem reasonable.

\subsection{Hypotheses}

Given the implications of prospect theory and the laws framed as protection- and reward-based incentive structures, I assume that people exhibit-in general-in a whistleblowing scenario a similarly s-shaped value function, that is convex (concave) for losses (gains). They evaluate outcomes in terms of gains and losses, with losses weighing stronger than gains of the same magnitude (loss aversion).

H 1.1 Participants exhibit an s-shaped value function, that is convex (concave) for losses (gains) in a whistleblowing scenario.

H 1.2 Participants exhibit pronounced loss aversion, which means that losses weigh heavier than gains of the same monetary (dis)value.

This general behavior should be irrelevant of the person's intention to whistleblow, since loss aversion is assumed to be a "general" behavioral trait in humans (Tversky and Kahneman 1992).

H 2 Whistleblowing intention is independent of loss aversion.

However, the application of prospect theory also implies that if losses weigh heavier than gains, then a protection from a loss, e.g., retaliation or sanction, should influence a whistleblower stronger than a reward of the same monetary value.

Not only loss aversion in prospect theory suggests that losses should have a stronger impact on people than gains, but also results of whistleblowing research discussed earlier. In particular, the many studies that suggest retaliation to have a strong influence on the whistleblowing intention and the findings by Andon et al. (2018) and Rose et al. (2018) in relation to the rather mixed influence of rewards substantiate the idea that losses weigh heavier than gains. Thus, a loss is a stronger dis-motivation to whistleblow, than a gain increases intention to whistleblow. 
H 3.1 A loss decreases whistleblowing intention, while a gain increases whistleblowing intention.

H 3.2 A loss has a stronger impact on intention than a gain in equal value magnitude.

\section{Experiment description}

The experiment was conducted in a university's experimental laboratory. Forty-six university students were chosen at random from a pool of almost 700 valid subjects. Due to strong violations in their answering pattern, 7 participants had to be excluded after the data collection, ${ }^{4}$ leaving a valid sample of 39 . The experiment aimed at eliciting their respective value function through a process of questions, using a method developed by Abdellaoui et al. (2016). Twenty-five participants of the original sample were invited for a second experiment, where they were asked for their whistleblowing intention in respect of their answers from the first experiment. One participant belonged to the excluded group, resulting in a second sample of 24 subjects. ${ }^{5}$ The participants' mean age was 22.74 (22.25) for the sample with 39 (24) students. They consisted of 48.7 (50) percent women and had on average participated in 31-35 (31-35) economic courses in the course of their studies.

For the purpose of this experiment, let $S$ be a set of states of nature and subsets of $S$ events $E$, where $1-E$ is the complement of $E$. $E$ is always assigned to the larger payoff, and $1-E$ to the smaller one. The events are weighted, just as probabilities would be, by a decision weight $W$, which may differ for gains and losses. $X$ is a set of consequences, also called outcomes. $X$ contains a neutral outcome $x_{0}$, and all other outcomes are monetary gain (loss) payoffs higher (lower) than $x_{0}$. There are pure gain- and loss-, as well as mixed prospects, with a maximum of 2 outcomes per prospect. A mixed prospect would therefore be depicted as $W^{+}(E) V\left(x^{+}\right)+$ $W^{-}(1-E) V\left(x^{-}\right)$, and a sure gain prospect would simply be $W^{+}(E) V\left(x^{+}\right)$where $W^{+}(E)=1$ and $E=1$; thus, $W^{+}(E) V\left(x^{+}\right)=V\left(x^{+}\right)$.

An overview of the experimental stages is given in Table 2. As a starting point (part 0), participants were given the short case description about the fraud they observed and that subsequent questions would show them several alternatives between which they would have to give values (in monetary terms) for which they are indifferent between the alternatives (prospects). The ambiguity in this context was also explained, as well as test questions to help participants get acquainted with the experimental design. A translation of the case and the description of ambiguity can be found in Appendix 2. The original case was repeatedly shown throughout the experiment to remind participants of the situation they were to imagine.

\footnotetext{
4 They often and early on in the experiment violated stochastic dominance and gave contradictory answers repeatedly.

5 The reason for splitting the sessions was the fear that a single session would take too long and thus lead to less reliable data. The reason for only inviting a subsample for the intention questions was due to budgetary restraints. However, results are highly significant despite the small sample sizes. Tversky and Kahnemann (1992), for example, used only 25 subjects in their entire sample.
} 
Table 2 Experimental stages and elicitation procedure following Abdellaoui et al. (2016); value elicited in monetary terms

\begin{tabular}{|c|c|c|c|c|}
\hline & & Value elicited (verbal) & Value elicited & Decision problem \\
\hline Part 0 & \multicolumn{4}{|c|}{ Explanation of case, ambiguity (uncertainty) context, alternatives, and test questions } \\
\hline \multirow[t]{3}{*}{ Part 1} & 1.1 & Loss $L_{1}$, given: gain $G=60,000$ EUR & $L_{1}$ & $x_{0} \sim G L_{1}$ \\
\hline & 1.2 & First positive outcome $x_{1}^{+}$ & $x_{1}^{+}$ & $G x_{0} \sim x_{1}^{+}$ \\
\hline & 1.3 & First negative outcome $x_{1}^{-}$ & $x_{1}^{-}$ & $L_{1} x_{0} \sim x_{1}^{-}$ \\
\hline \multirow[t]{2}{*}{ Part 2} & 2.1 & Loss $L_{2}$, given: loss $l=9000$ EUR & $L_{2}$ & $l x_{0} \sim x_{1}^{+} L_{2}$ \\
\hline & 2.2 & Second to sixth positive outcome $x_{j}^{+} ; j \in\{2, \ldots, 6\}$ & $x_{j}^{+}$ & $x_{j-1}^{+} l \sim x_{j}^{+} L_{2}$ \\
\hline \multirow[t]{3}{*}{ Part 3} & 3.1 & Gain & $G_{1}$ & $g x_{0} \sim G_{1} x_{1}^{-}$ \\
\hline & & $G_{1}$, given: gain $g=9000$ EUR & & \\
\hline & 3.2 & Second to sixth negative outcome $x_{j}^{-} ; j \in\{2, \ldots, 6\}$ & $x_{j}^{-}$ & $g x_{j-1}^{-} \sim G_{1} x_{j}^{-}$ \\
\hline Part 4 & & Fourth outcome $x_{4}^{+}$(again, as control) & $x_{4}^{+}$ & $x_{3}^{+} l \sim x_{4}^{+} L_{2}$ \\
\hline Part 5 & & Demographic questions & \multicolumn{2}{|c|}{ Gender, age, lectures taken,... } \\
\hline
\end{tabular}

Each stage of the experiment consisted of two prospects. One outcome in one of the prospects was not given. For this outcome, or payoff, the participant had to decide on the monetary value for which she would be indifferent between the two given prospects. In several stages, losses $\left(L_{j}, l, x_{j}^{-}\right)$and gains $\left(G, g, x_{j}^{+}\right)$were given or to be set by the participant. Table 2 gives an overview. For example, in part 1.1 the two prospects $x_{0}$ and $G ; L_{1}$ were presented. $x_{0}$ is a sure prospect, while $G ; L_{1}$ is an ambiguous prospect, where either $G$ (a gain) or $L_{1}$ (a loss) realizes. This is given as $x_{0} \sim G L_{1}$. The payoff value for $L_{1}$ was to be elicited, meaning that the participant had to decide for which payoff $L_{1}$ she would be indifferent between the prospect $G ; L_{1}$ and the sure outcome $x_{0}$. Mathematically, this can be written as $W(1) V\left(x_{0}\right)=W^{+}(E) V\left(x^{+}\right)+W^{-}(1-E) V\left(x^{-}\right)$, where $x^{+}$is $G$ and $x^{-}$is $L_{1}$ and $W(1)=1$. Recall that in prospect theory the probability, or in this case the ambiguity, is weighted by a decision weight $W$, which may differ between positive and negative outcomes, thus $W^{+}$and $W^{-}$, respectively. Each elicitation took four stages. In the first, an estimate of the monetary outcome was elicited by giving seven outcome options spaced in equal difference. This starting value for the outcome was then used to calculate and present seven refined options in the second stage. In the third, the participant was given a range based on her previous answers in which a value had to be entered manually. The fourth stage showed the two prospects again with the elicited payoff inserted. Participants had to confirm that they were now indifferent between the two prospects. If they were not indifferent, the procedure would start again in stage 1. This procedure is based on the experiments by Tversky and Kahneman (1992) and Abdellaoui et al. (2016). An example of this procedure is given in Appendix 3.

As shown in Table 2, part 1 elicits the first gain and loss outcome, respectively, and part 2 (3) elicits the gains (losses) two to six. The corresponding value function of the outcomes is a ratio scale, in which each subsequent outcome has the same value difference. Without imposing any parametric restrictions, I can measure the 
Fig. 4 Normalized value function over all subjects' average elicited gain and loss outcomes

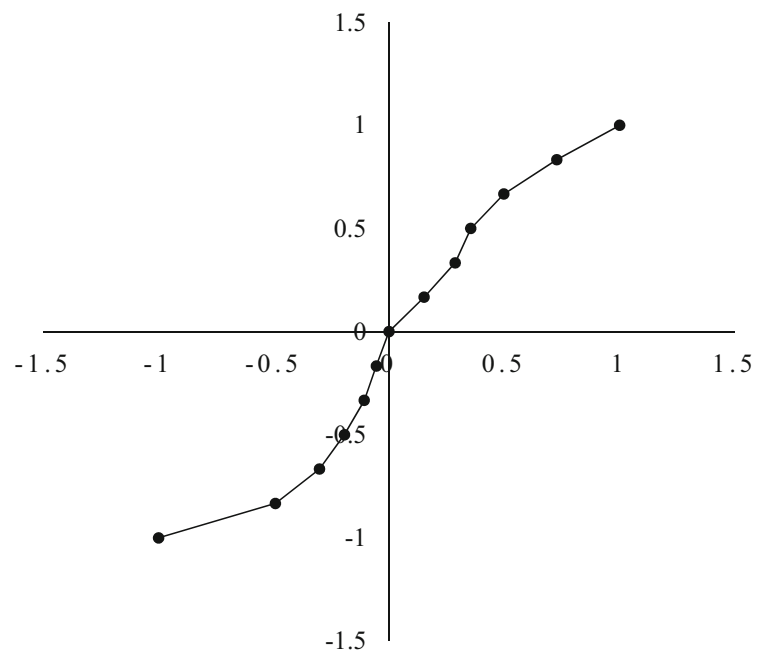

entire value function through the reference point. Why this holds is formally shown in Appendix 4. It mainly utilizes the work by Abdellaoui (2000) and Abdellaoui et al. (2016), who develop this method to make the value function observable for risk and ambiguity.

In part 4, subjects were asked to elicit a payoff value from part 2 again to control for inconsistency in the answering pattern. The Wilcoxon test (Wilcoxon 1945) shows no significant difference ( $p=.795$, two-sided). There is high correlation (Kendall's tau $.859, p<.001$ and Spearman's rho .962, $p<.001$ ) between both elicitations, indicating that both times the elicited values did not differ and therefore the decision pattern is indeed consistent. This can be seen as a validation of the procedure used in this experiment (Abdellaoui et al. 2016).

\section{Results}

\subsection{Value curvature}

The value function in prospect theory is s-shaped: For positive (negative) outcomes, it is concave (convex). To measure the curvature of the function, $v(x)$ can be normalized to 1 by scaling $V\left(x_{6}^{+}\right)=1\left(V\left(x_{6}^{-}\right)=-1\right)$ and for any other value $\mathrm{V}$ $\left(x_{j}^{+}\right)=j / 6\left(\mathrm{~V}\left(x_{j}^{-}\right)=-j / 6\right)$ for all $j \in\{1, \ldots .5\}$ (Abdellaoui et al. 2016). ${ }^{6}$ As an example, the mean function over all subjects normalized is shown in Fig. 4 and without normalization in Fig. 5. When Fig. 5 is compared to Fig. 3, one can already see that the shape is very similar to that of the typical value function proposed in prospect theory.

\footnotetext{
${ }^{6}$ This can only be done if $-x_{j}^{-} / x_{6}^{-}$and $x_{j}^{+} / x_{6}^{+}$iff $x_{j}^{-} \geq x_{6}^{-}$and $x_{j}^{+} \leq x_{6}^{+}$(Abdellaoui et al. 2016).
} 


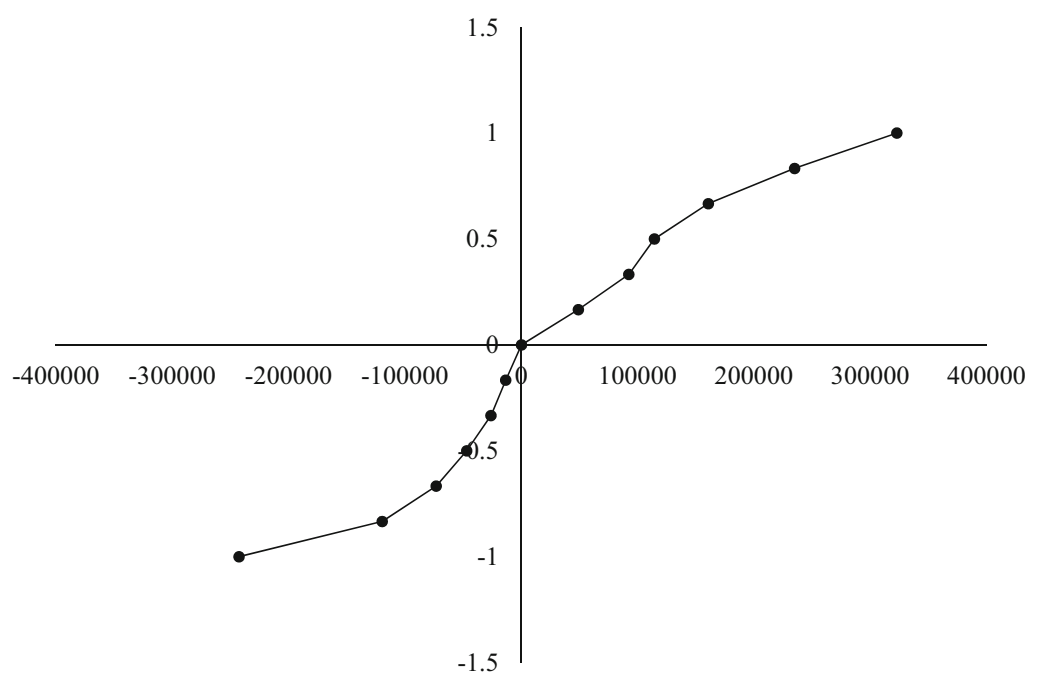

Fig. 5 Value function over all subjects' average elicited outcomes

The area under the normalized curve for gains (losses) can more accurately be measured and interpreted for each subject individually as $\int_{0}^{1} f\left(x^{+}\right) \mathrm{d} x\left(-\int_{-1}^{0} f\left(x^{-}\right) \mathrm{d} x\right)$, where an area $<.5$ indicates a convex (concave),$=.5$ linear (linear), and $>.5$ concave (convex) shape. The results are shown in Table 3. Almost half of the subjects $(46.2 \%)$ can be classified according to prospect theory's s-shaped value function, while only $15.4 \%$ acted according to the traditional idea of constant risk aversion. Almost $70 \%$ changed their risk preferences at the reference point, further validating prospect theory's idea of reference dependence. Results are similar to previous studies (Tversky and Kahneman 1992; Abdellaoui et al. 2016).

This can be seen as a confirmation of hypothesis 1.1 that participants would exhibit behavioral traits consistent with prospect theory in terms of the shape of the value function: It is s-shaped for a majority of subjects (marked in bold) and almost half of the students show concave (convex) value functions in the area of gains (losses).

\subsection{Loss aversion}

Several ways to measure loss aversion have emerged over the years. The original method proposed by Kahneman and Tversky (1979) (K/T) assumes loss aversion when $V(x)<-V(-x)$ for all $x>0$. Since $V\left(x_{j}^{+}\right)=-V\left(-x_{j}^{-}\right), x_{j}^{+}>-x_{j}^{-}$ implies that $V\left(x_{j}^{+}\right)<-V\left(-x_{j}^{+}\right)$holds. This can be measured using a Wilcoxon test for all $x_{j}$ (Table 4).

The difference is significant for all $x_{j}^{+}>\left|x_{j}^{-}\right|$, and the effect is very pronounced $(r=|Z / \sqrt{n}|>.5$ for all $Z$, is considered a strong effect, while $>.3$ is considered a 
Table 3 Value curvature shape of the participants, where concave gain and convex loss curves are in line with prospect theory

\begin{tabular}{lllll}
\hline & Losses & & & \\
\cline { 2 - 5 } & Concave & Convex & Linear & Total \\
\hline Gains & & & & \\
Concave & 6 & $\mathbf{1 8}$ & 0 & 24 \\
Convex & 7 & 0 & 0 & 11 \\
Linear & 2 & 22 & 2 & 4 \\
Total & 15 & 2 & $\mathbf{3 9}$ \\
\hline
\end{tabular}

Table 4 Wilcoxon difference test between gains and losses for all elicited outcomes

\begin{tabular}{lrrrrrr}
\hline Wilcoxon test & $-x_{1}^{-} ; x_{1}^{+}$ & \multicolumn{1}{c}{$-x_{2}^{-} ; x_{2}^{+}$} & \multicolumn{1}{c}{$-x_{3}^{-} ; x_{3}^{+}$} & $-x_{4}^{-} ; x_{4}^{+}$ & $-x_{5}^{-} ; x_{5}^{+}$ & $-x_{6}^{-} ; x_{6}^{+}$ \\
\hline$Z$ based on positive ranks & -5.106 & -3.916 & -3.256 & -2.900 & -3.181 & -2.454 \\
Asymptotic significance & $.000^{* * *}$ & $.000^{* * *}$ & $.001^{* *}$ & $.4^{* *}$ & $.001^{* *}$ & $.014^{*}$ \\
two-sided & & & & & & \\
\hline
\end{tabular}

*** Significant on a level of $p<.001 ; * *$ significant on a level of $p<.01$;* Significant on a level of $p<.05$

medium effect and $>.1$ is a small effect according to Cohen 1988). Loss aversion can be assumed to be present based on the K/T measure (Kahneman and Tversky 1979).

Another measurement was introduced by Tversky and Kahneman (1992) (T/K) by calculating the loss aversion parameter $\lambda$ :

$$
v(x)\left\{\begin{array}{cc}
x_{j}^{\alpha} & x \geq 0 \\
-\lambda\left(-x_{j}\right)^{\beta} & x<0
\end{array} .\right.
$$

Given that $\alpha$ and $\beta$ are found to be equal across many studies (see Bromiley 2010 for a review), then positive outcomes (ordinate) divided over the absolute values of the negative outcomes (abscissa) for the respective $x_{j}$ make $\lambda$ observable with $x_{j}^{+}=\lambda\left(-x_{j}^{-}\right)$. This also holds because $V(x)$ is a ratio scale with known values (Abdellaoui et al. 2016). A function can be elicited by linear interpolation with the origin as reference and starting point of the function, shown in Fig. 6, where $\lambda=2.5336$. This is in line with prior studies who found $\lambda$ to be between 2.3 (Abdellaoui et al. 2016) and 2.83 (Baltussen et al. 2016) for ambiguity. For risk, Tversky and Kahneman (1992) elicited a value of 2.25. Other studies found values for risk of 2.47 (Abdellaoui et al. 2011), 2.21 (Abdellaoui et al. 2016), and 1.86 (Baltussen et al. 2016). This deviation between the values for ambiguity and risk may be attributed to the effect found by Ellsberg (1961) that people in general exhibit higher aversion toward uncertainty over probability. Thus, pronounced loss aversion can be assumed to be present based on the $\mathrm{T} / \mathrm{K}$ measure. 
Fig. 6 Loss aversion elicitation according to the $T / K$ test for $\lambda$, where the dashed line is the linearly interpolated function for loss aversion and the dotted line is a function of $y=x$ for reference, that is if $\lambda$ were 1

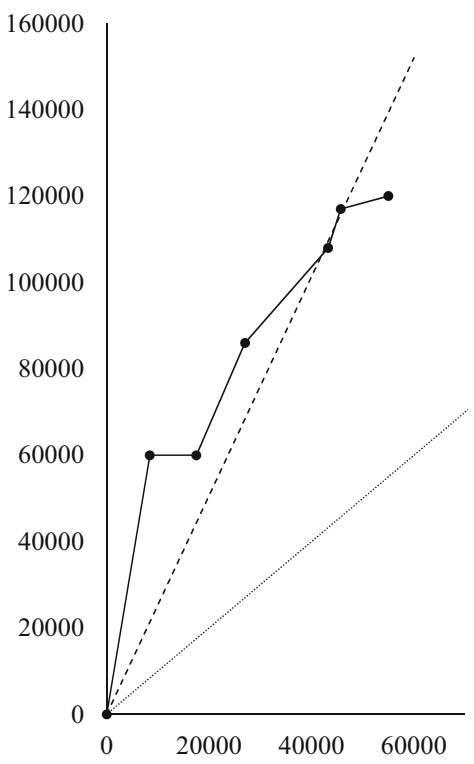

A third method proposed by Köbberling and Wakker (2005) (K/W) measures the kink at the reference point, thus the left derivative over the right derivative at the reference point as $V_{\uparrow}^{\prime}(0) / V_{\downarrow}^{\prime}(0)$. Abdellaoui et al. (2016) simplify this assumption by arguing that the fist gain and loss payoffs elicited may be used. Then $\lambda=$ $V_{\uparrow}^{\prime}(0) / V_{\downarrow}^{\prime}(0)=V\left(x_{1}^{-}\right) / x_{1}^{-} / V\left(x_{1}^{+}\right) / x_{1}^{+}$and since $V\left(x_{1}^{-}\right)=-V\left(x_{1}^{+}\right)$, one can simply calculate $x_{1}^{+} /-x_{1}^{-}$. Doing this individually for all 39 subjects, the median (mean) value is 4.7619 (9.5858). Using the median (mean) values for $x_{1}$ over all subjects for gains and losses, respectively, gives a loss aversion of 7.1429 (3.6527). These values are arguably higher than the one produced by the $\mathrm{T} / \mathrm{K}$ method. A simple explanation is that the K/W method only takes into account the initial elicited values of the function in either direction, whereas the $T / K$ method measures the entire function. The latter is thus less influenced by outliers and seems more robust. Regardless of method used, participants in general exhibit strong loss aversion. A loss weighs more than twice as heavy as a gain of the same absolute monetary value. This is a confirmation of the hypothesis 1.2 that people exhibit strong loss aversion. Hypotheses 1.1 and 1.2 show that the reference point is important for people to value payoffs in terms of gains and losses in a law context. The results suggest that this holds for high payoffs and ambiguity.

\subsection{Whistleblowing intention}

A subsample of the first group $(n=24)$ was invited to participate in a second part of the experiment. Here, the intention to whistleblow was measured, using five questions $\left(i n t_{j}\right.$ ) that asked for the intention to actually come forward with the information in different ways. This is a normal procedure often used in 
whistleblowing research (Park et al. 2008; Park and Blenkinsopp 2009; Brown, Hays and Stuebs 2016). A 7-point Likert scale $(1, \ldots, 7)$ was used, where 1 equals "I do not agree at all" and 7 "I fully agree." The translated questions and their descriptive statistics are given in Table 5.

The questions showed very high internal consistency (Cronbach's alpha $=.911$ ), indicating that the questions actually measured the same underlying aspect. The same case as in the first part of the experiment was given. Each participant was subsequently confronted with her alternatives from the first test, and her (individually elicited) payoffs. For each alternative the participant had to score on a 7-point Likert scale her intention to actually whistleblow, given each prospect. As an example, recall part 1.1 of Table 2 . Two prospects were given: $x_{0}$ and $G ; L_{1}$. The elicited value for $L_{1}$ from the first experiment was inserted and the participant had to state her intention to whistleblow given the prospect $x_{0}$ and her intention for the situation where either $G$ or $L_{1}$ realizes.

The correlation for the overall intention to whistleblow with loss aversion was identified, using the individual loss aversion parameters $\mathrm{W} / \mathrm{K}$ and $\mathrm{T} / \mathrm{K}$ elicited in the first experiment for each subject and their overall whistleblowing intention (int sum $_{\text {) }}$ measured as the sum of each participant's int ${ }_{j}$ answer. As shown in Table 6 there is no significant correlation between the intention and loss aversion for either measures $(p>.05)$, indicating that these are separate concepts and not influenced by each other.

This is in line with hypothesis 2 that assumed whistleblowing intention and behavior according to prospect theory, namely loss aversion, to be separate concepts. However, intention should nonetheless change according to the "laws" of prospect theory. That is, a loss should decrease intention to whistleblow stronger than a gain would increase it, as proposed by hypotheses $\mathrm{H} 3.1$ and 3.2.

Next, the change of intention to whistleblow is measured when moving along the value function. Since the value difference (recall that $V(x)$ is a ratio scale and that the intention to whistleblow was elicited for each prospect) between subsequent outcomes is equal, it is possible to compare the intention at given points along the value function. This also holds for changes since equal changes in magnitude along the value function can be measured. For reasons of simplicity the value for the observed loss (gain) is set to -1 (1). The value at the reference point is 0 by definition (Tversky and Kahneman 1992). Thus, a change from the reference point

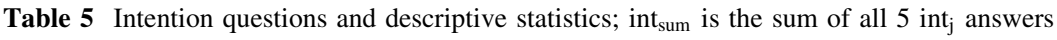

\begin{tabular}{llrrr}
\hline Item & Statement & Mean & St.d. & Var. \\
\hline int $_{1}$ & I intend to report the case externally. & 5.25 & 1.42 & 2.02 \\
int $_{2}$ & I will report the case externally. & 4.71 & 1.33 & 1.78 \\
int $_{3}$ & I am willing to report the case externally. & 5.33 & 1.49 & 2.23 \\
int $_{4}$ & I plan to report the case externally. & 5.42 & 1.35 & 1.82 \\
int $_{5}$ & I believe it is my duty to report the case externally. & 5.13 & 1.62 & 2.64 \\
int $_{\text {sum }}$ & & 25.83 & 6.22 & 38.67 \\
\hline
\end{tabular}

1 Equals "I do not agree at all" and 7 equals "I fully agree" on a 7-point Likert scale. St.d. = standard deviation; var. = variance; internal consistency: Cronbach's alpha $=.911$ 
Table 6 Intention and loss aversion measures correlation using rank-sum tests' Kendall's tau and Spearman's rho

\begin{tabular}{|c|c|c|c|c|}
\hline $\begin{array}{l}\text { Rank-sum } \\
\text { test }\end{array}$ & $\begin{array}{l}\text { Variable for } \\
n=24\end{array}$ & Measure & $\begin{array}{l}\text { Loss aversion measure: } \\
\mathrm{W} / \mathrm{K}\end{array}$ & $\begin{array}{l}\text { Loss aversion } \\
\text { measure: } \mathrm{T} / \mathrm{K}\end{array}$ \\
\hline \multirow[t]{2}{*}{$\begin{array}{l}\text { Kendall's } \\
\text { tau }\end{array}$} & int $_{\text {sum }}$ & $\begin{array}{l}\text { Correlation } \\
\text { coefficient }\end{array}$ & -.289 & -.099 \\
\hline & & $\begin{array}{l}\text { Significance } \\
\text { two-sided }\end{array}$ & .057 & .514 \\
\hline \multirow[t]{2}{*}{$\begin{array}{l}\text { Spearman's } \\
\text { rho }\end{array}$} & int $_{\text {sum }}$ & $\begin{array}{l}\text { Correlation } \\
\text { coefficient }\end{array}$ & -.399 & -.153 \\
\hline & & $\begin{array}{l}\text { Significance } \\
\text { two-sided }\end{array}$ & .053 & .475 \\
\hline
\end{tabular}

***Significant at a level of $p>.001$;**significant at a level of $p<.01$; *significant at a level of $p<.05$; int $_{\text {sum }}$ is the sum of the 5 individual int $_{j}$ values

in either direction is in magnitude equal to 1 as well. Intention at the reference point is given as int $\mathrm{ref}_{\text {, for the sure loss (gain) as int }}$ ( (int $_{\text {pos }}$ ), and for the uncertain loss (gain) as int neg_unc $_{\text {(int }}$ pos_unc $)$. The intention change was calculated when moving from the reference point (prospect: sure $x_{0}$; part 1.1) to a sure loss (prospect: sure

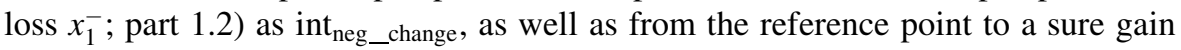

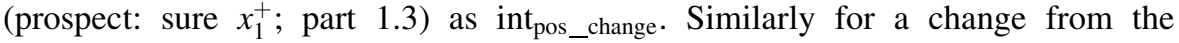
reference point to an uncertain loss (gain), prospect: 1.3 (prospect: 1.2), as int $_{\text {neg_unc_change }}\left(\right.$ int $\left._{\text {pos_unc_change }}\right)$. These positions and changes in intention to whistleblow were then evaluated using a Wilcoxon test. Table 7 shows the comparisons of positions in intention.

Intention to whistleblow changes significantly with a change of the outcome from the reference point toward a sure loss (gain). However, the effect is stronger for a change toward a loss $(r=.81)$ than a gain $(r=.52)$. This effect realizes although the monetary value for a gain is already on average more than twice as high as the absolute value of the loss. The change toward an uncertain outcome from the reference point is only significant for losses, not for gains. The change from a certain to an uncertain outcome (recall that the value of the outcome remains at 1) is only significant for losses, not for gains. This can be interpreted as that an uncertain loss increases whistleblowing intention compared to a certain loss, whereas whistleblowing intention is not significantly increased by an uncertain gain toward a certain one, keeping the value of the outcome constant.

Table 8 shows the comparison of changes in intention. There are significant differences between an equal (dis)value change toward the domain of gains and losses from the reference point. An equal value change from the reference point to a loss is felt significantly stronger than the one from the reference point to a gain (int neg_change $_{\text {vs int }}$ pos_change). The effect is strong $(r=.51)$ and realizes although the absolute magnitude of the change is 1 , and the value function already controls for the person's loss aversion. The impact on intention of a change from the reference point toward a sure loss is found to be significantly stronger than toward an uncertain loss, but this effect is not significant for gains. Instead of direct intention 
Table 7 Intention position differences using a Wilcoxon test

\begin{tabular}{llllllrl}
\hline $\begin{array}{l}\text { Wilcoxon } \\
\text { test variables }\end{array}$ & $\begin{array}{l}\text { int }_{\text {pos }} ; \\
\text { int }_{\text {ref }}\end{array}$ & $\begin{array}{l}\text { int }_{\text {neg }} ; \\
\text { int }_{\text {ref }}\end{array}$ & $\begin{array}{l}\text { int }_{\text {pos_unc }} ; \\
\text { int }_{\text {ref }}\end{array}$ & $\begin{array}{l}\text { int }_{\text {neg_unc }} ; \\
\text { int }_{\text {ref }}\end{array}$ & $\begin{array}{l}\text { int }_{\text {pos }} ; \\
\text { int }_{\text {unc_pos }}\end{array}$ & $\begin{array}{l}\text { int }_{\text {neg }} ; \\
\text { int }_{\text {unc_neg }}\end{array}$ & \multicolumn{1}{c}{$\begin{array}{l}\text { int }_{\text {neg }} ; \\
\text { int }_{\text {pos }}\end{array}$} \\
\hline$Z$ & $-2.549^{\mathrm{a}}$ & $-3.946^{\mathrm{b}}$ & $-1.794^{\mathrm{a}}$ & $-2.524^{\mathrm{b}}$ & $-1.730^{\mathrm{a}}$ & $-2.217^{\mathrm{b}}$ & $-4.087^{\mathrm{b}}$ \\
$\begin{array}{l}\text { Asymptotic } \\
\text { significance, } \\
\text { two-sided }\end{array}$ & $.011^{*}$ & $.000^{* * *}$ & .073 & $.012^{*}$ & .084 & $.027^{*}$ & $.000^{* * *}$ \\
\hline
\end{tabular}

${ }^{\mathrm{a} B a s e d}$ on negative ranks

${ }^{\mathrm{b}}$ Based on positive ranks

***Significant at a level of $p<.001$; **significant at a level of $p<.01$; *significant at a level of $p<.05$

Table 8 Intention changes differences using a Wilcoxon test

\begin{tabular}{lll}
\hline Wilcoxon test variables & $Z$ & Asymptotic significance 2-sided \\
\hline int $_{\text {neg_change }}-$ int $_{\text {pos_change }}$ & $-2.508^{\mathrm{a}}$ & $.012^{*}$ \\
int $_{\text {pos_change }}-$ int $_{\text {pos_unc_change }}$ & $-1.730^{\mathrm{a}}$ & .084 \\
int $_{\text {neg_unc_change }}-$ int $_{\text {neg_change }}$ & $-2.217^{\mathrm{b}}$ & $.027^{*}$ \\
\hline
\end{tabular}

${ }^{\mathrm{a}}$ Based on negative ranks

${ }^{\mathrm{b}}$ Based on positive ranks

$* * *$ Significant at a level of $p<.001 ; * *$ significant at a level of $p<.01$; *significant at a level of $p<.05$

changes, the percentage change was calculated as a control. For example, the percentage change from 5 to 7 is less than one from 2 to 4 , where the value doubles. The untabulated analysis gives the same results. The asymptotic significance for the difference between an uncertain and certain gain are even less pronounced ( $p=.115 ; Z=-1.577$ based on positive ranks).

Hypothesis 3.1 stated that a loss would decrease whistleblowing intention, while a gain would increase whistleblowing intention. This can partially be shown with the analysis above. Deviations toward losses (gains) decrease (increase) whistleblowing intention, but there is no difference in the intention between a sure gain and an uncertain gain, whereas there is a significant difference for the loss side.

Hypothesis 3.2 stated that a loss has a stronger impact on intention than a gain of the same magnitude. Intention to whistleblow is decreased stronger by a loss than it is increased by a gain. Figure 7 illustrates this again graphically for the mean intention positions, where the intentions for the (uncertain) loss and gain are depicted. It can be seen that the impact on the loss side for a decrease in intention is much stronger than the increase in intention for a gain. This is despite the fact that for the same absolute value of the outcome $(v(x)=1)$, the monetary gain on average is already more than twice as high as the absolute loss.

\section{Discussion}

The initial research question was one of applicability of prospect theory and its value as a prescriptive instrument in economics. As an application example, legislation on whistleblowing was used, which is often framed as either promoting 


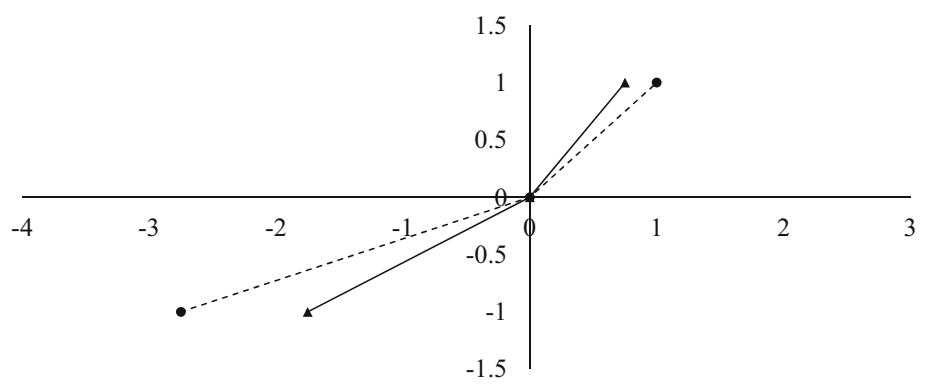

Fig. 7 Average intention change for certain (dashed line with dots) and uncertain (straight line with triangles) outcomes

rewards or as a prevention of losses, e.g., from sanction or retaliation by protecting whistleblower. In addition, prospect theory was found to be underutilized in its application for ambiguity and high-stake contexts.

The results imply that prospect theory explains behavior in situations under ambiguity and high (hypothetical) payoffs well. The majority of participants showed the typical s-shaped value function, with concave (convex) shape for gains (losses) that changes at the reference point (reference dependence). Participants exhibited loss aversion in a magnitude similar to prior studies for ambiguity. These findings further validate the applicability of prospect theory outside of traditional lottery game frames (Tversky and Kahneman 1992). Limitations obviously stem from the experimental nature of this research. In addition, the measure of whistleblowing intention was previously criticized for not adequately capturing actual whistleblowing (Mesmer-Magnus and Viswesvaran 2005), a general issue that is hard to rectify (Miceli and Near 1984). Although participants were only asked to evaluate the monetary outcomes, they may just as well have interpreted the sanctions with additional losses, such as job loss, retaliation by friends and co-workers, and other negative outcomes, influencing their answers in the experiment.

Nonetheless, results strongly suggest that potential whistleblowers evaluate legislation within a prospect theory frame. Their intention to whistleblow and their loss aversion are not correlated, meaning that this evaluation is independent of intention. However, their intention changes when faced with different prospects. They react stronger to losses than to gains in their intention to whistleblow, where a loss results in a stronger change to not come forward than a similar change toward a gain incentivizes whistleblowing. This effect realizes despite the fact that the monetary value of the gain is on average already twice as high as the absolute loss outcome. Furthermore, a sure gain does not increase the intention over an uncertain gain significantly. A certain loss over an uncertain one, on the other hand, influences the intention to whistleblow: A certain loss decreases whistleblowing intention stronger than an uncertain loss.

This has several implications for whistleblowing legislation. Uncertain rewards, such as the SEC pays whistleblower (SEC 2018), have less of an impact on the intention to whistleblow than a protection-even if it does not protect whistleblower for sure-an unrealistic assumption in general, given that cases usually are decided in court rooms (Moberly 2007). 
To address the first research question of whether and how prospect theory may be applied, let me return to the initial Tversky and Kahneman (1992, p. 137) reference: "rationality in economic theory is commonly justified [...] [by] the fear that any treatment that abandons rationality will be chaotic and intractable. [...] the evidence indicates that human choices are orderly, although not always rational in the traditional sense of this word." Although human behavior may not be fully rational, it is predictable. This may be utilized in order to construct effective legislation. Any law that aims at incentivizing or discouraging certain behavior may use this insight. If outcomes can be framed as gains and losses from a reference point, reducing the impacts on the loss side should be given priority-or increase of the loss side, in case of discouragement. Barberis (2013) urges to "use the insights of prospect theory in a more prescriptive way: to nudge people toward behaviors that are viewed as more desirable" (Barberis 2013, p. 190). Prospect theory may be applied to current issues not only in a way that explains behavior but more prescriptively, encourages or prevents behavior. I have shown that this can be applied on current issues such as the proposed legislative efforts by the European Union. It lends economic credibility to an otherwise morally grounded reasoning for a law that would protect but not reward whistleblowing. This is not to say that legal literature does not already utilize this logic, but it mainly fails to ground it in a comprehensive theoretical framework such as prospect theory.

The impact of such a legislation especially for whistleblowing may have its limits. As previous research shows, whistleblowing intention is influenced by several factors in a complex way (Chiu 2003; Brown et al. 2014). In particular, culture may influence the decision and practitioners should evaluate regulative efforts carefully. A European law as proposed by the European Commission should, given the results of this experiment, be an adequate solution in fostering whistleblowing. However, such a one-size-fits-all approach comes at the expense of taking into account individual, situational, and cultural differences in countries (Park et al. 2008, p. 937: "the relation between cultural orientation and attitudes toward whistleblowing cannot be generalized across countries"; Brown et al. 2014; Cheng et al. 2015). This is an aspect that needs further evaluation.

Although hard law was used to show that prospect theory can be applied to real and current issues, the results can be applied to several other contexts. For example, compliance management systems, reward structures for management, and agents of control, such as the auditing profession, may benefit from these implications. Companies reward short-term or even self-centered incentivized behavior by management, e.g., with bonuses, that may in the long term not only harm the company and thus the shareholder value, but also the public. They may find themselves outnumbered by whistleblowers who are willing to speak up when comprehensive protection is added to mandatory compliance systems, that are already in place in many companies. Also, companies need not worry as much about laws such as the DFA that rewards external whistleblowing (Schmidt 2005), when an internal whistleblowing system adds efficient protection for people willing to come forward with information.

Future research should explore more avenues for the applicability of prospect theory. There seems much potential for legal research, which has so far mostly ignored this theory. Experiments involving practitioners in the field, such as 
auditors, could result in more valuable insight into the effectiveness of compliance management and monitoring systems. On a theoretical basis, more research should utilize prospect theory as an instrument for decisions under ambiguity, since most real-world decisions involve not only risk but uncertainty on many levels.

\section{Conclusion}

The initial research question was one of applicability of prospect theory and its value as a prescriptive instrument in economics. As an application example, legislation on whistleblowing was used, which is often framed as legislation either favoring rewards or the prevention of losses by protecting whistleblower. In addition, prospect theory was found to be underutilized in its application for ambiguous and high-stake contexts.

In an experiment with 39 university students I find that participants affirm the assumptions of prospect theory. That is, they exhibit a typical s-shaped value function which is concave (convex) for gains (losses) and changes at the reference point (reference dependence). Participants evaluate outcomes in terms of gains and losses, where losses weigh heavier than gains (loss aversion). In addition, whistleblowing intention is independent of loss aversion, which implies that the value function and its implications are general behavioral traits. However, whistleblowing intention changes with different prospects in a way prospect theory suggests. Whistleblowing intention decreases more for changes toward losses than it increases by changes toward gains of the same value magnitude from a reference point. This is despite the fact that the monetary value of the gain is already twice as high as the absolute loss. An uncertain loss provides higher whistleblowing intention than a certain one, whereas there is no change in intention between a certain gain and an uncertain one. This can be attributed to the evaluation in terms of gains and losses with pronounced loss aversion irrespective of a person's general loss aversion. Effective whistleblowing legislation should therefore focus rather on reducing the effects on the loss side, e.g., protection from retaliation, than on reward structures.

More generally, I show that prospect theory may be used in a prescriptive way, utilizing people's "irrational", yet predictable behavior in order to design effective legislation. This logic may be applied across a range of different issues, not only in the domain of hard law, but also for compensation structures, compliance management and control systems or for discouraging certain behavior.

Acknowledgements I am very grateful to Prof. Dr. Anne Chwolka, participants of the Business and Economics Faculty Colloquium of Otto-von-Guericke University, as well as three anonymous reviewers for very helpful comments.

Open Access This article is distributed under the terms of the Creative Commons Attribution 4.0 International License (http://creativecommons.org/licenses/by/4.0/), which permits unrestricted use, distribution, and reproduction in any medium, provided you give appropriate credit to the original author(s) and the source, provide a link to the Creative Commons license, and indicate if changes were made. 


\section{Appendix 1}

\section{See Table 9.}

Table 9 Country law texts

\begin{tabular}{ll}
\hline Country & Whistleblowing law/provision \\
\hline Australia & Public Interest Disclosure Act 2013 \\
Belgium & $\begin{array}{c}\text { Law on Reporting a Suspected Integrity } \\
\text { Violation in a Federal Administrative } \\
\text { Authority by a Staff Member }\end{array}$ \\
European & $\begin{array}{c}\text { Proposal for a directive of the European } \\
\text { Union }\end{array}$ \\
& Parliament and of the Council on the \\
& protection of persons reporting on \\
breaches of Union law & \\
France & SAPIN II Act on transparency \\
Hungary & Act on Complaints and Public Interest \\
Ireland & Disclosures
\end{tabular}

Italy

Luxembourg Law on Strengthening the Means to Fight Corruption

Malta

Protection of the Whistleblower Act

Netherlands House for Whistleblowers Act

Norway Norway, Working Environment Act 2005 (as amended in 2017)

Section 2 A-1 The right to notify censurable conditions at the undertaking

Romania

Slovakia

Slovenia

Sweden

The UK
Law on the Protection of Public Officials Complaining about Violations of the Law

Act on Certain Measures related to the Reporting of the Anti-social Activities and on amendments of certain laws

Only partially in the Integrity and Prevention of Corruption Act

Act on Special Protection Against Victimization of Workers who Sound the Alarm on Serious Wrongdoing

Public Interest Disclosure Act (PIDA)
Online

http://www.comlaw.gov.au/Details/ C2013A00133

https://www.whistleblower-rights.org/ whistleblower-protection-laws-3/

https://eur-lex.europa.eu/legal-content/EN/ TXT/?uri=CELEX:52018PC0218

www.legifrance.gouv.fr/eli/loi/2016/12/9/

ECFM1605542L/ jo\#JORFARTI000033558655

https://www.whistleblower-rights.org/ whistleblower-protection-laws-3/

The full text of Ireland Protected Disclosures Act 2014 is available at www.irishstatutebook.ie/eli/2014/act/14/ enacted/en/html

https://www.whistleblower-rights.org/ whistleblower-protection-laws-3/

https://www.whistleblower-rights.org/ whistleblower-protection-laws-3/

https://www.whistleblower-rights.org/ whistleblower-protection-laws-3/

https://www.whistleblower-rights.org/ whistleblower-protection-laws-3/

An English translation of Norway, Working Environment Act is available at www. arbeidstilsynet.no/contentassets/ e54635c3d2e5415785a4f23f5b852849/ working-environment-act-october-web2017.pdf

https://www.whistleblower-rights.org/ whistleblower-protection-laws-3/

The full text of the law (in Slovak) can be found at www.slov-lex.sk/pravnepredpisy/SK/ZZ/2014/307/20160701

https://www.whistleblower-rights.org/ whistleblower-protection-laws-3/

https://www.whistleblower-rights.org/ whistleblower-protection-laws-3/

https://www.legislation.gov.uk/ukpga/1998/ $23 /$ contents 
Table 9 continued

\begin{tabular}{|c|c|c|}
\hline Country & Whistleblowing law/provision & Online \\
\hline The USA & $\begin{array}{l}\text { Sarbanes-Oxley Act } 18 \text { U.S.C. } \S 1514 \text { A } \\
\text { (SOX)/Dodd-Frank Wall Street Reform } \\
\text { and Consumer Protection Act } 15 \text { U.S.C. } \\
\text { §78u-6 Section } 922 \text { (DFA) }\end{array}$ & $\begin{array}{l}\text { https://www.gpo.gov/fdsys/pkg/FR-2015- } \\
\text { 03-05/html/2015-05001.htm and: https:// } \\
\text { www.gpo.gov/fdsys/pkg/PLAW- } \\
\text { 111publ203/html/PLAW-111publ203. } \\
\text { htm }\end{array}$ \\
\hline
\end{tabular}

\section{Appendix 2: Case and ambiguity description}

The original case description was in German. This is a loose translation by the author.

Imagine you work for an international company in the accounting department. In the course of your daily work you find some peculiar documents that might point toward a case of fraud, in which senior management might be involved, should your thoughts be correct. To report the case internally is therefore not an option for you. You report externally.

Ambiguity description:

The realization probabilities for a specific outcome within an alternative are unknown. You cannot know if the probability for the first outcome within an alternative is $1 \%, 50 \%$ of $99 \%$. However, since one of the two outcomes has to realize, you know that if the likelihood for the first outcome is $30 \%$, then the likelihood for the second outcome is $70 \%$, the adverse probability.

Most accurately, the uncertainty within an alternative can be described as follows: The probability for the first outcome is larger than $0 \%$, but smaller than $100 \%$. And the probability for the second outcome is the remaining probability (adverse probability).

However, the probability for a sanction and the probability for a reward remain constant over all alternatives in the course of the experiment.

\section{Appendix 3: First 3 stages of a sample elicitation process}

The original cases were presented in German. This is a loose translation by the author. 
1. At which monetary value for the sanction in alternative 2 are you indifferent between the two alternatives? [S101]

altermative 1: No change of your situation.

vs.

alternative 2: Reward of 60.000 Euro or sanction of __ Euro.
20.000
30.000
40.000
50.000
60.000
70.000
80.000

2. At which monetary value for the sanction in alternative 2 are you indifferent between the two alternatives?

Here you can refine your answer. [S102]

-

altermative 1: No change of your situation.

vs.

alternative 2: Reward of 60.000 Euro or sanction of Euro.
51000
54000
57000
60000
63000
66000
69000

3. At which monetary value for the sanction in alternative 2 are you indifferent between the two alternatives? [S103]

Your selection was between $\mathbf{6 0 0 0 0}$ and $\mathbf{6 6 0 0 0}$. Choose the monetary value that most accurately captures your preference within the spread.

alternative 1: No change of your situation.

vs.

alternative 2: Reward of 60.000 Euro or sanction of 62500 Euro. 


\section{Appendix 4: Value elicitation}

The elicitation procedure is mainly based on Abdellaoui (2000) and Abdellaoui et al. (2016).

$$
\begin{aligned}
& \text { Part } 1.1: x_{0} \sim G L_{1} \\
& W^{+}(E) V(G)+W^{-}(1-E) V\left(L_{1}\right)=V\left(x_{0}\right)=0 \\
& \text { Part 1.2: } G x_{0} \sim x_{1}^{+} \text {and } L_{1} x_{0} \sim x_{1}^{-} \text {, then: } \\
& \text { Part } 1.3: V\left(x_{1}^{+}\right)=W^{+}(E) V(G) \\
& V\left(x_{1}^{-}\right)=W^{-}(1-E) V\left(L_{1}\right) \text { thus: } \\
& V\left(x_{1}^{+}\right)=-V\left(x_{1}^{-}\right) \\
& \text {Part 2.1:l } l x_{0} \sim x_{1}^{+} L_{2} \text {, then : } \\
& W^{+}(E) V\left(x_{1}^{+}\right)+W^{-}(1-E) V\left(L_{2}\right)=W^{-}(1-E) V(l)+V\left(x_{0}\right) \text { with } V\left(x_{0}\right)=0 \\
& V\left(x_{1}^{+}\right)-V\left(x_{0}\right)=\frac{W^{-}(1-E)}{W^{+}(E)}\left(V(l)-V\left(L_{2}\right)\right) \\
& \text { Part } 2.2: x_{1}^{+} l \sim x_{2}^{+} L_{2} \text {, then : } \\
& V\left(x_{2}^{+}\right)-V\left(x_{1}^{+}\right)=\frac{W^{-}(1-E)}{W^{+}(E)}\left(V(l)-V\left(L_{2}\right)\right)
\end{aligned}
$$

Equations 1.9 and 1.11 yield:

$$
V\left(x_{2}^{+}\right)-V\left(x_{1}^{+}\right)=V\left(x_{1}^{+}\right)-V\left(x_{0}\right)
$$

Thus, a sequence for gains $\left\{x_{0}, x_{1}^{+}, \ldots, x_{6}^{+}\right\}$can be elicited, where subsequent $x_{j}^{+}$ have the same value difference, which means that $V(x)$ can be written as a value scale with equally spaced values:

$$
\begin{gathered}
\text { For all } x_{j-1}^{+} l \sim x_{j}^{+} L_{2} \text { with } j \in(2, \ldots, 6): \\
V\left(x_{j}^{+}\right)-V\left(x_{j-1}^{+}\right)=V\left(x_{1}^{+}\right)-V\left(x_{0}\right)
\end{gathered}
$$

This is done similarly for part 3 and the outcome elicitation of losses:

Part 3.1: $g x_{0} \sim G_{1} x_{1}^{-}$to obtain $G_{1}$ to elicit a sequence of losses:

Part 3.2: $\left\{x_{0}, x_{1}^{-}, x_{2}^{-}, \ldots, x_{6}^{-}\right\}$for all $g x_{j-1}^{-} \sim G_{1} x_{j}^{-}$where $j \in(2, \ldots, 6)$

Thus, all outcomes $\left\{x_{6}^{-}, \ldots, x_{1}^{-}, x_{0}, x_{1}^{+}, \ldots, x_{6}^{+}\right\}$have equal value differences to their successive outcome through the reference point. 


\section{References}

Abdellaoui, M. 2000. Parameter-Free Elicitation of Utility and Probability Weighting Functions. Management Science 46(11): 1497-1512.

Abdellaoui, M., H. Bleichrodt, O. L'Haridon, and D. van Dolder. 2016. Measuring Loss Aversion under Ambiguity: A Method to Make Prospect Theory Completely Observable. Journal of Risk and Uncertainty 52(1): 1-20.

Abdellaoui, M., O. L'Haridon, and C. Paraschiv. 2011. Experienced vs. Described Uncertainty: Do we Need Two Prospect Theory Specifications? Management Science 57(10): 1879-1895.

Andon, P., C. Free, R. Jidin, G.S. Monroe, and M.J. Turner. 2018. The Impact of Financial Incentives and Perceptions of Seriousness on Whistleblowing Intention. Journal of Business Ethics 151(1): 165-178.

Association of Certified Fraud Examiners (ACFE). 2016. Report to the Nations on Occupational Fraud and Abuse. Texas: Austin.

Ball, R. 2009. Market and Political/Regulatory Perspectives on the Recent Accounting Scandals. Journal of Accounting Research 47(2): 277-323.

Baltussen, G., M.J. van den Assem, and D. van Dolder. 2016. Risky Choice in the Limelight. The Review of Economics and Statistics 98(2): 318-332.

Barberis, N.C. 2013. Thirty Years of Prospect Theory in Economics: A Review and Assessment. Journal of Economic Perspectives 27(1): 173-196.

Bowden, P. 2006. A Comparative Analysis of Whistleblower Protections. Australian Journal of Professional and Applied Ethics 8(2): 1-14.

Brennan, N. and J. Kelly. 2007. A Study of Whistleblowing Among Trainee Auditors. The British Accounting Review 39(1): 61-87.

Brink, A.G., D.J. Lowe, and L.M. Victoravich. 2017. The Public Company Whistleblowing Environment: Perceptions of a Wrongful Act and Monetary Attitude. Accounting and the Public Interest 17(1): $1-30$.

Bromiley, P. 2010. Looking at Prospect Theory. Strategic Management Journal 31: 1357-1370.

Brown, A.J., M.T. Rehg, T. Uys, and W. Vandekerckhove. 2014. Understandings of Whistleblowing: Dilemmas of Societal Culture. In International Handbook of Whistleblowing Research, ed. A.J. Brown, D. Lewis, R. Moberly, and W. Vandekerckhove, 37-70. Cheltenham: Edward Elgar.

Brown, J.O., J. Hays, and M.T. Stuebs. 2016. Modeling Accountant Whistleblowing Intentions: Applying the Theory of Planned Behavior and the Fraud Triangle. Accounting and the Public Interest 16(1): 28-56.

Bussmann, K.-D. 2015. The Impact of Personality and Company Culture on Company Anti-corruption Programs. In Routledge Handbook of White-Collar and Corporate Crime in Europe, ed. W. Huisman and G. Vande Walle, 435-452. Halle, Germany: PricewaterhouseCoopers AG and Martin-LutherUniversity Halle-Wittenberg.

Bussmann, K.-D., C. Nestler, and S. Salvenmoser. 2013. Wirtschaftskriminalität und Unternehmenskultur 2013. Halle.

Caillier, J.G. 2017. An Examination of the Role Whistle-Blowing Education Plays in the WhistleBlowing Process. The Social Science Journal 54: 4-12.

Call, A.C., G.S. Martin, N.Y. Sharp, and J.H. Wilde. 2018. Whistleblowers and Outcomes of Financial Misrepresentation Enforcement Actions. Journal of Accounting Research 56(1): 123-171.

Callahan, E.S. and T.M. Dworkin. 1994. Who Blows the Whistle to the Media and Why: Organizational Characteristics of Media Whistleblowers. American Business Law Journal 151: 153-158.

Camerer, C.F. 1989. An Experimental Test of Several Generalized Utility Theories. Journal of Risk and Uncertainty 2: 61-104.

Charreire Petit, S. and J. Cusin. 2013. Whistleblowing and Resilience: Analysis of an Individual Trajectory.M@n@gement 16(2): 141-175.

Cheng, X., K. Karim, and K.J. Lin. 2015. A Cross-Cultural Comparison of Whistleblowing Perceptions. International Journal of Management and Decision Making 14(1): 15-31.

Cherry, B., L.D. Ordóñez, and S.W. Gilliland. 2003. Grade Expectations: The Effects of Expectations on Fairness and Satisfaction Perceptions. Journal of Behavioral Decision Making 16(5): 375-395.

Chiu, R.K. 2003. Ethical Judgment and Whistleblowing Intention: Examining the Moderating Role of Locus of Control. Journal of Business Ethics 43(1-2): 65-74.

Cohen, J. 1988. Statistical Power Analysis for the Behavioral Sciences. Hillsdale: Erlbaum. 
Cordis, A.S. and E.M. Lambert. 2017. Whistleblower Laws and Corporate Fraud: Evidence from the United States. Accounting Forum 41: 289-299.

Dhamija, S. and S. Rai. 2017. Role of Retaliation and Value Orientation in Whistleblowing Intentions. Asian Journal of Business Ethics 7(1): 37-52.

Dworkin, T.M. 2007. SOX and Whistleblowing. Michigan Law Review 105(8): 1757-1780.

Dworkin, T.M. and M.S. Baucus. 1998. Internal vs. External Whistleblowers: A Comparison of Whistleblowing Processes. Journal of Business Ethics 17(1998): 1281-1298.

Dyck, A., A. Morse, and L. Zingales. 2010. Who Blows the Whistle on Corporate Fraud? The Journal of Finance 65(6): 2213-2253.

Ellsberg, D. 1961. Risk, Ambiguity and the Savage Axioms. Quarterly Journal of Economics 75: 643-669.

Ethics Research Center. 2016. Global Business Ethics Survey (GBES) (2016). Measuring Risk and Promoting Workplace Integrity. Arlington: Ethics \& Compliance Initiative.

European Commission. (2018). Proposal for a Directive of the European Parliament and of the Council on the Protection of Persons Reporting on Breaches of Union Law: COM(2018) 218 - 2018/0106 (COD). http://ec.europa.eu/newsroom/just/item-detail.cfm?item_id=620400. Accessed 1 Sept 2018.

Fennema, H. and P. Wakker. 1997. Original and Cumulative Prospect Theory: a Discussion of Empirical Differences. Journal of Behavioral Decision Making 10(1): 53-64.

Gao, L. and A.G. Brink. 2017. Whistleblowing Studies in Accounting Research: A Review of Experimental Studies on the Determinants of Whistleblowing. Journal of Accounting Literature 38: $1-13$.

Gibeaut, J. 2006. Culture Clash: Other Countries Don't Embrace Sarbanes or America's Reverence of Whistle-Blowers. ABA Journal 92(5): 10-12.

Grant, C. 2002. Whistle Blowers: Saints of Secular Culture. Journal of Business Ethics 39(2002): 391-399.

Gray, J.A. 2004. The Scope of Whistleblower Protection in the State of Maryland: A Comprehensive Statute Is Needed. University of Baltimore Law Review 33(2): 225-256.

Hartman, L., D. Elm, T. Radin, and K.R. Pope. 2009. Translating Corporate Culture Around the World: A Cross-Cultural Analysis of Whistleblowing as an Example of How to Say and Do the Right Thing. notizie di POLITEIA XXV(93): 255-272.

Hershey, J.C. and P.J.H. Schoemaker. 1980. Prospect Theory's Reflection Hypothesis: A Critical Examination. Organizational Behavior and Human Performance 25: 395-418.

Hirschman, A.O. 1970. Exit, Voice and Loyalty. Responses to Decline in Firms, Organizations and States. Cambridge: Harvard University Press.

Holmes, R.M., P. Bromiley, C.E. Devers, T.R. Holcomb, and J.B. McGuire. 2011. Management Theory Applications of Prospect Theory: Accomplishments, Challenges, and Opportunities. Journal of Management 37(4): 1069-1107.

Kachelmeier, S.J. and M. Shehata. 1992. Examining Risk Preferences Under High Monetary Incentives: Experimental Evidence from the People's Republic of China. The American Economic Review 82(5): 1120-1141.

Kahneman, D. and A. Tversky. 1979. Prospect Theory: An Analyses of Decision Under Risk. Econometrica 47(2): 263-292.

Keenan, J.P. 2002. Comparing Indian and American Managers on Whistle-Blowing. Employee Responsibilities and Rights Journal 14(2): 79-89.

Keenan, J.P. 2007. Comparing Chinese and American Managers on Whistleblowing. Employee Responsibilities and Rights Journal 19(2): 85-94.

Knight, F.H. 1921. Risk, Uncertainty, and Profit. Boston: Houghton Mifflin.

Köbberling, V. and P.P. Wakker. 2005. An Index of Loss Aversion. Journal of Economic Theory 122: $119-131$.

Köszegi, B. and M. Rabin. 2007. Reference-Dependent Risk Attitudes. The American Economic Review 97(4): 1047-1073.

Kothiyal, A., V. Spinu, and P.P. Wakker. 2014. An Experimental Test of Prospect Theory for Predicting Choice Under Ambiguity. Journal of Risk and Uncertainty 48(1): 1-17.

Latimer, P. and A.J. Brown. 2008. Whistleblower Laws: International Best Practice. UNSW Law Journal 31(3): 766-794.

Lee, G. and X. Xiao. 2018. Whistleblowing on Accounting-Related Misconduct: A Synthesis of the Literature. Journal of Accounting Literature (forthcoming). 
Li, X. and I. McNeil. 2006. Comply or Explain: Market Discipline and Non-compliance with the Combined Code. Corporate Governance: An International Review 14(5): 486-496.

Liyanarachchi, G. and C. Newdick. 2009. The Impact of Moral Reasoning and Retaliation on WhistleBlowing: New Zealand Evidence. Journal of Business Ethics 89(1): 37-57.

Mayer, D.M., S. Nurmohamed, L. Klebe Treviño, D.L. Shapiro, and M. Schminke. 2013. Encouraging Employees to Report Unethical Conduct Internally: It Takes a Village. Organizational Behavior and Human Decision Processes 121: 89-103.

Mesmer-Magnus, J.R. and C. Viswesvaran. 2005. Whistleblowing in Organizations: An Examination of Correlates of Whistleblowing Intentions, Actions, and Retaliation. Journal of Business Ethics 62(2005): 277-297.

Miceli, M.P. and J.P. Near. 1984. The Relationships among Beliefs, Organizational Position, and WhistleBlowing Status: A Discriminant Analysis. The Academy of Management Journal 27(4): 687-705.

Moberly, R. 2007. Unfulfilled Expectations: An Empirical Analysis of why Sarbanes-Oxley Whistleblowers Rarely Win. William and Mary Law Review 49: 65-155.

Mogielnicki, M. 2011. Hunting for 'Bounty' and Finding 'Moral Autonomy': The Dodd-Frank Act Expansion of Whistle Blower Protections. Academy of Business Research 2: 74-84.

Near, J.P. and M.P. Miceli. 1985. Organizational Dissidence: The Case of Whistle-Blowing. Journal of Business Ethics 4(1985): 1-16.

Near, J.P. and M.P. Miceli. 1996. Whistle-Blowing: Myth and Reality. Journal of Management 22(3): $507-526$.

Park, H. and J. Blenkinsopp. 2009. Whistleblowing as Planned Behavior-A Survey of South Korean Police Officers. Journal of Business Ethics 85(4): 545-556.

Park, H., J. Blenkinsopp, M.K. Oktem, and U. Omurgonulsen. 2008. Cultural Orientation and Attitudes Toward Different Forms of Whistleblowing: A Comparison of South Korea, Turkey, and the U.K. Journal of Business Ethics 82(2008): 929-939.

Perreault, S. and J. Wainberg. 2016. Whistleblowing in Audit Firms: Do Explicit Protections from Retaliation Activate Implicit Threats of Reprisal? Behavioral Research in Accounting 28(1): 83-93.

Rauhofer, J. 2007. Blowing the Whistle on Sarbanes-Oxley: Anonymous Hotlines and the Historical Stigma of Denunciation in Modern Germany. International Review of Law Computers and Technology 21(2007): 363-376.

Rose, J.M., A.G. Brink, and C.S. Norman. 2018. The Effects of Compensation Structures and Monetary Rewards on Managers' Decisions to Blow the Whistle. Journal of Business Ethics 150(3): 853-862.

Samuelson, W. and R. Zeckhauser. 1988. Status Quo Bias in decision Making. Journal of Risk and Uncertainty 1(1): 7-59.

Schmidt, M. 2005. Whistle Blowing Regulation and Accounting Standards Enforcement in Germany and Europe-An Economic Perspective. International Review of Law and Economics 25(2): 143-168.

SEC (Securities and Exchange Commission). 2018. 2017 Annual Report to Congress: Whistleblower Program. Washington, DC: SEC.

Sims, R.L. and J.P. Keenan. 1999. A Cross-Cultural Comparison of Managers' Whistleblowing Tendencies. International Journal of Value- Based Management 12(2): 137-151.

Stikeleather, B.R. 2016. When Do Employers Benefit from Offering Workers a Financial Reward for Reporting Internal Misconduct? Accounting, Organizations and Society 52: 1-14.

Tversky, A. and D. Kahneman. 1981. The Framing of Decisions and the Psychology of Choice. Science 211(4481): 453-458.

Tversky, A. and D. Kahneman. 1992. Advances in Prospect Theory: Cumulative Representation of Uncertainty. Journal of Risk and Uncertainty 5(4): 297-323.

Villena, M.G. and M.J. Villena. 2010. On the Economics of Whistle-Blowing Behavior: The Role of Incentives. Munich Personal RePEc Archive Paper Nr. 36310: 1-28.

Vinten, G. 2004. Whistleblowing: The UK experience, Part 2. Management Decision 42(1-2): 139-151.

Weingarten, E., S. Bhatia., and B. Mellers. 2018. Multiple Goals as Reference Points: One Failure Makes Everything Else Feel Worse. Management Science (forthcoming).

Wilcoxon, F. 1945. Individual Comparisons by Ranking Methods. Biometrics Bulletin 1(6): 80-83.

Wilde, J.H. 2017. The Deterrent Effect of Employee Whistleblowing on Firms' Financial Misreporting and Tax Aggressiveness. The Accounting Review 92(5): 247-280.

Yang, C., B. Liu, L. Zhao, and X. Xu. 2017. An Experimental Study on Cumulative Prospect Theory Learning Model of Travelers' Dynamic Mode Choice Under Uncertainty. International Journal of Transportation Science and Technology 6(2): 143-158. 
Yeoh, P. 2014. Whistleblowing: Motivations, Corporate Self-Regulation, and the Law. International Journal of Law and Management 56(6): 459-474.

Publisher's Note Springer Nature remains neutral with regard to jurisdictional claims in published maps and institutional affiliations. 